\title{
miR824/AGAMOUS-LIKE16 Module Integrates Recurring Environmental Heat Stress Changes to Fine-Tune Poststress Development
}

\author{
Henrik Mihály Szaker 1,2, Éva Darkó ${ }^{3}$, Anna Medzihradszky ${ }^{1}$, Tibor Janda ${ }^{3}$, \\ Hsiang-chin Liu ${ }^{4}$, Yee-yung Charng ${ }^{4}$ and Tibor Csorba ${ }^{1 *}$ \\ ${ }^{1}$ Agricultural Biotechnology Institute, NARIC, Godollo, Hungary, ${ }^{2}$ Faculty of Natural Sciences, Eötvös Lóránd University, \\ Budapest, Hungary, ${ }^{3}$ Agricultural Institute, Centre for Agricultural Research, Hungarian Academy of Sciences, Martonvásár, \\ Hungary, ${ }^{4}$ Agricultural Biotechnology Research Center, Academia Sinica, Taipei, Taiwan
}

OPEN ACCESS

Edited by:

László Szabados, Hungarian Academy of Sciences, Hungary

Reviewed by: Juan José Ripoll, University of California, San Diego, United States Jie Le, Institute of Botany (CAS), China

*Correspondence: Tibor Csorba csorba.tibor@abc.naik.hu

Specialty section: This article was submitted to Plant Abiotic Stress, a section of the journal

Frontiers in Plant Science

Received: 02 August 2019 Accepted: 18 October 2019 Published: 25 November 2019

Citation:

Szaker HM, Darkó É, Medzihradszky A, Janda T, Liu H-C, Charng Y-y and Csorba T (2019) miR824/AGAMOUS-LIKE16 Module Integrates Recurring Environmental Heat Stress Changes to Fine-Tune Poststress Development.

Front. Plant Sci. 10:1454. doi: 10.3389/fp/s.2019.01454
Plant development is continually fine-tuned based on environmental factors. How environmental perturbations are integrated into the developmental programs and how poststress adaptation is regulated remains an important topic to dissect. Vegetative to reproductive phase change is a very important developmental transition that is complexly regulated based on endogenous and exogenous cues. Proper timing of flowering is vital for reproductive success. It has been shown previously that AGAMOUS LIKE 16 (AGL16), a MADS-box transcription factor negatively regulates flowering time transition through FLOWERING LOCUS T (FT), a central downstream floral integrator. AGL16 itself is negatively regulated by the microRNA miR824. Here we present a comprehensive molecular analysis of miR824/AGL16 module changes in response to mild and recurring heat stress. We show that miR824 accumulates gradually in response to heat due to the combination of transient transcriptional induction and posttranscriptional stability. miR824 induction requires heat shock cis-elements and activity of the HSFA1 family and HSFA2 transcription factors. Parallel to miR824 induction, its target AGL16 is decreased, implying direct causality. AGL16 posttranscriptional repression during heat stress, however, is more complex, comprising of a miRNA-independent, and a miR824-dependent pathway. We also show that $A G L 16$ expression is leaf vein-specific and overlaps with miR824 (and FT) expression. AGL16 downregulation in response to heat leads to a mild derepression of $F T$. Finally, we present evidence showing that heat stress regulation of miR824/AGL16 is conserved within Brassicaceae. In conclusion, due to the enhanced post-transcriptional stability of miR824, stable repression of AGL16 is achieved following heat stress. This may serve to fine-tune FT levels and alter flowering time transition. Stress-induced miR824, therefore, can act as a "posttranscriptional memory factor" to extend the acute impact of environmental fluctuations in the poststress period.

Keywords: AGAMOUS-LIKE16, miR824, heat stress, posttranscriptional memory factor, FLOWERING LOCUS T 


\section{INTRODUCTION}

In nature, plants adapt to the diurnally and seasonally fluctuating environment for successful growth and reproduction. Heat stress (HS) is one of the most important abiotic stresses. Plant HS response (HSR) is triggered by a number of temperature sensing pathways (Mittler et al., 2012; Yeh et al., 2012). One important task of the HSR signal transduction pathways is the activation of HS transcription factors (HSFs) (Mittler et al., 2012; Scharf et al., 2012; Yeh et al., 2012). Multiple HSFs are involved in basal HSR, HSFA1 family transcription factors (in Arabidopsis HSFAla, HSFA1b, HSFA1d, and HSFA1e) being its master regulators (Liu et al., 2011; Yoshida et al., 2011). HSFA1 paralogs, among others, turn on the transcription of HSFA2 (Charng et al., 2007; NishizawaYokoi et al., 2011; Liu and Charng, 2012; Liu and Charng, 2013). HSFA1s, together with HSFA2 induce the expression of various types of heat shock proteins (HSPs) and nonchaperone proteins (Scharf et al., 2012). When plants encounter HS for the first time, they become acclimated (primed). Under natural conditions, acclimation occurs gradually during the day and repeatedly at the beginning of the hot season. This so-called acquired thermotolerance allows plants to survive upcoming stronger, even lethal stresses (Mittler et al., 2012; Lamke and Baurle, 2017; Liu et al., 2018). Active maintenance of acquired thermotolerance for several days after the stress is the HS memory. HSFA2 is the central component of HS memory (Charng et al., 2007; Lamke et al., 2016; Liu et al., 2018). HS memory also requires the chromatin remodeling factor FORGETTER1 (Brzezinka et al., 2016), the chromatin-associated protein BRUSHY1/TONSOKU/ MGOUN3 (Brzezinka et al., 2018), the HSP HEAT-STRESSASSOCIATED32 (HSA32)(Charng et al., 2006; Wu et al., 2013), a peptidyl cis/trans isomerase ROTAMASE FKBP1(Meiri and Breiman, 2009) and HSFA1s or HSFA1-related factors (Liu et al., 2018). While the different forms of HSR have been intensively studied, how plants integrate sporadic or repeated stress signals and alter their development following stress is much less known.

MicroRNAs (miRNAs) are an important class of small RNAs, the central players of RNA silencing (Axtell, 2013; Rogers and Chen, 2013; Borges and Martienssen, 2015). miRNAs are encoded by distinct genes, transcribed by RNA polymerase II. miRNA transcripts may contain introns, therefore they undergo splicing. Subsequent to the splicing, the fold-back structures of miRNA precursors (pri-miRNAs) are maturated by DICERLIKE proteins in two steps to give rise to the pre-miRNA and the mature miRNA duplex. The mature miRNAs are loaded into ARGONAUTE proteins, the effector of silencing, to form RNA-induced silencing complex (RISC). In plants, RISC cleaves destabilizes or represses translation of its target messenger RNAs (mRNAs) guided by the nucleotide sequence of the loaded miRNA (Chen, 2004; Brodersen et al., 2008; Rogers and Chen, 2013; Borges and Martienssen, 2015). miRNAs regulate developmental and metabolic processes like cell differentiation, organ development, senescence, hormonal biosynthesis, nutrient uptake, and allocation (Schommer et al., 2008; Rubio-Somoza and Weigel, 2011; Matthewman et al., 2012; Luo et al., 2013; Li and Zhang, 2016). miRNAs are also involved in responses to environmental changes (Sunkar et al., 2012; Guan et al., 2013;
Cui et al., 2014; Kruszka et al., 2014; Kumar, 2014; Zhang, 2015). Several miRNAs were shown to be heat-responsive in numerous species including Arabidopsis thaliana, Brassica rapa, Populous euphratica, Triticum aestivum, Oryza sativa, and others (Xin et al., 2011; Chen et al., 2012; Yu et al., 2012; Barciszewska-Pacak et al., 2015; Kumar et al., 2015; Mangrauthia et al., 2017; Gyula et al., 2018). In a few cases, the activity of stress-regulated miRNAs was studied in details and the precise molecular function unraveled (Guan et al., 2013; Cui et al., 2014; Stief et al., 2014; Ma et al., 2015; He et al., 2018).

MADS-box containing proteins are a large class of eukaryotic transcriptional factors involved in diverse pathways like development and environmental interactions (Yanofsky et al., 1990; Messenguy and Dubois, 2003; Smaczniak et al., 2012). In plants, MADS-box proteins play central and conserved roles in cell differentiation of the embryo, the gametophyte and the vegetative tissue development, the transition to flowering, the flower organogenesis, and fruit ripening (Yanofsky et al., 1990; Ferrandiz et al., 2000a; Ferrandiz et al., 2000b; Liljegren et al., 2000; Pelaz et al., 2000; Nesi et al., 2002; Michaels et al., 2003; De Bodt et al., 2005; Tao et al., 2012; Csorba et al., 2014; Fernandez et al., 2014; Whittaker and Dean, 2017). Several MADS-box proteins were shown to be stress-regulated (Lozano et al., 1998; Arora et al., 2007; Tardif et al., 2007; Saha et al., 2015; Chen et al., 2016). AGAMOUS-LIKE16 (AGL16) is a $\mathrm{MIKC}^{\mathrm{C}}$-type MADS-box protein-coding gene, a member of the AGL17 clade (Smaczniak et al., 2012). The genes of the AGL17 clade are primarily expressed in roots suggesting rootrelated functions (Alvarez-Buylla et al., 2000; Burgeff et al., 2002; Gan et al., 2005). AGL16 is expressed more ubiquitously: besides root, it was found also in the stem and rosette leaves, inflorescence, and young siliques (Alvarez-Buylla et al., 2000). A miRNA, namely the miR824, negatively regulates $A$. thaliana AGL16 (Kutter et al., 2007; Hu et al., 2014). Two functions of AGL16 have been described in detail so far. Mutation of AGL16 or overexpression of miR824 decreased the number of higherorder stomata complexes, while the expression of miR824resistant AGL16 in transgenic plants increased the incidence of higher-order stomata complexes (Kutter et al., 2007). The miR824/AGL16 module was also shown to modulate flowering time in A. thaliana under long-day conditions (Hu et al., 2014; McClung et al., 2016). The AGL16 protein interacts directly with the SHORT VEGETATIVE PHASE protein and indirectly with FLOWERING LOCUS C (FLC). FLC is a central regulator of flowering transition (Lee and Amasino, 1995; McClung et al., 2016; Whittaker and Dean, 2017). The FRIGIDA (FRI) complex drives high expression of FLC (Geraldo et al., 2009; Choi et al., 2011). The negative regulatory effect of AGL16 on the flowering time is fully dependent on the repression of FLOWERING LOCUS T (FT) (Hu et al., 2014; RomeraBranchat et al., 2014). rsa-miR824, the Raphanus sativus homolog of miR824, was also linked to flowering regulation (Nie et al., 2015) suggesting functional conservation of the module. The presence of AGL16-like proteins in all the investigated plant species so far, suggests that they play important roles in angiosperm development and evolution (Becker and Theissen, 2003; Gramzow and Theissen, 2015). 
In this work, we gather evidence suggesting that miR824 may act as an integrator of repeated HS signals to modulate AGL16 levels. As the impact of miR824-dependent AGL16 downregulation is primarily manifested poststress, miR824 may act as a posttranscriptional stress memory factor to alter development through fine-tuning FT pathway in response to environmental changes. We also show that heat-mediated regulation of the miR824/ AGL16 module is conserved in multiple members of Brassicaceae.

\section{MATERIALS AND METHODS}

\section{Plant Material and Growth Conditions}

Arabidopsis seeds were bleach-sterilized, stratified for 2 days in dark then plated on Murashige and Skoog (Duchefa M0222, https://www.duchefa-biochemie.com) medium agar plates (0.5 $\mathrm{x}$ Murashige and Skoog salts, $1 \%$ agar, $\mathrm{pH}$ 5.7). Plants were routinely grown in a Sanyo MLR-350 growth cabinet under cool white light at $21^{\circ} \mathrm{C}$ long day condition (16 h light $/ 8 \mathrm{~h}$ dark photoperiod).

\section{Heat Stress Treatments}

ACC: Gradient acclimation was done in the presence of light in a water bath in the course of $4 \mathrm{~h}$ : the temperature was rose starting at Zeitgeber Time $\mathrm{ZT} 4$ and reached $37^{\circ} \mathrm{C}$ at ZT7; plants were kept on $37^{\circ} \mathrm{C}$ for $1 \mathrm{~h}$ from ZT7 to ZT8. Plants were cooled back to $21^{\circ} \mathrm{C}$ following each treatment. Single ACC treatment was done on day 6 , in case of two acclimations (ACCx2) on days 5 and 6 , in case of three acclimations (ACCx3) on days 4, 5, and 6 postgermination. Samples were taken on day 6 immediately after treatment for ACC samples, or the next day at ZT8 for REC samples.

HS: for direct HS naïve 7 day old seedlings grown on agar plates were exposed to $45^{\circ} \mathrm{C}$ in a water bath in the presence of light for $30 \mathrm{~min}$ and samples collected immediately at midday (ZT8). ACCx3 + HS: seedlings were first acclimated as described for ACCx 3 then exposed to HS. $37^{\circ} \mathrm{Cx} 3$ : seedlings were exposed to direct $37^{\circ} \mathrm{C}$ in a water bath in the presence of light for $1 \mathrm{~h}$ at ZT7 to ZT8, then cooled back to $21^{\circ} \mathrm{C}$. Treatments were done on days 4, 5, and 6 postgermination. Samples were taken at ZT8.

For FT measurements seedlings were heat-treated at $37^{\circ} \mathrm{C}$ for $1 \mathrm{~h}$ each (from ZT7 to ZT8) at days 7, 8, and 9. Samples were taken at the end of the light period (ZT16) on day 10 (Hu et al., 2014) to allow recovery of AGL16 in Col-FRI; $\triangle 824$ plants.

\section{Accession Numbers of Genes Used in the Study}

miR824 (At4g24415), miR398a (At2g03445), U6 (At3g14735), AGL16 (At3g57230), ACT2 (At3g18780), PP2a (At1g69960), HSFA1a (At4g17750), HSFA1b (At5g16820), HSFA1d (At1g32330), HSFA1e (At3g02990), HSFA2 (At2g26150), HSFA3 (At5g03720), HSFA6a (At5g43840), HSFA6b (At3g22830), HSFA7a (At3g51910), HSFA7b (At3g63350), FRIGIDA (At4g00650), FLC (At5g10140), Bna-miR824 (LOC106440800), BnaAGL16 (LOC106357131), BnaPP2A5 (LOC106382560), XRN4 (At1g54490), SKI2 (At3g46960), CSD1 (At1g08830), PP2AA3 (At1g13320), RD29A (At5g52310), UBC22 (At5g05080).

\section{Mutant and Transgenic Lines Used in the Study}

agl16-1 (SALK_104701) (Kutter et al., 2007), $\Delta 824$ (SALK_138988), MIM824 [line 12 from (Hu et al., 2014)], aTK, $b T K, d T K, e T K$, and QK (Liu et al., 2011), hsfa2 (SALK_008978) (Charng et al., 2007), hsfa3 (SALK_011107) (Schramm et al., 2008), hsfa6a (SALK_089880) (Hwang et al., 2014), hsfa6b (GK_513_ A02) (Huang et al., 2016), hsfa7a (WiscDsLox318F08), hsfa7b (SALK_152004) (Charng et al., 2007), xrn4-6 (SALK_014209) (Gy et al., 2007), ski2-2 (SALK_129982) (Branscheid et al., 2015), flc-2 (Michaels and Amasino, 1999).

\section{Genotyping}

Genomic DNA was extracted with extraction buffer [100 mM glycine, $10 \mathrm{mM}$ ethylenediaminetetraacetic acid (EDTA), $100 \mathrm{mM} \mathrm{NaCl}, 2 \%$ sodium dodecyl sulfate (SDS)] at room temperature, purified with phenol:chloroform: isoamyl alcohol (25:24:1) $\mathrm{pH}$ 8.0, precipitated in ethanol and resuspended in sterile water. Genotyping PCR was done using DNA Taq polymerase (NEB, M0273S) based on manufacturer instructions. For primer sequences see Primer Table.

\section{Transgene Constructs}

For miR824-promoter GUS transgenic lines, the 2,954 bp fragment comprising the $2,852 \mathrm{bp}$ sequences upstream of the transcription start site (TSS, +1) and 102 bp segment downstream of TSS was amplified in a PCR reaction (Phiuson, Thermo Scientific) cloned into the pGEM-T-easy vector (Promega). For mutant promoter generation PCR mutagenesis was done using mutagen primers (for sequences see primer Table) (wt HSE1: gTTCtaGAAc, mutant HSE1: gTCtaGAc, wt HSE-like 2: cTTCaaaGAAt, mutant HSE-like 2: cTTaaaAAt, wt HSE-like 3: aTTCaaGGAg, mutant HSE-like 3: aTTaaGAg), and then fused with the GUS reporter gene in pCAMBIA1301 at EcoRI and NcoI sites following elimination of $35 \mathrm{~S}$ promoter region. After sequencing the inserted fragments to confirm the absence of mutations caused by PCR and the presence of sitedirected mutations introduced in the mutant HSE promoters, independent transgenic lines were generated in Col-0 via floral dipping using C58C1 Agrobacterium strain (Clough and Bent, 1998). Plants were selected on hygromycin and confirmed as positives in Northern blot and GUS activity assays. The first rosette leaf of $\mathrm{T} 1$ seedlings or mature leaves, stems, and inflorescence of T1 plants was used for Northern blotting, qRTPCR or GUS staining assays. Northern blotting, qRT-PCR assays, and GUS staining were done in at least three biological replicates if not stated otherwise.

For pAGL16::GUS reporter construct promoter sequence of AGL16 gene comprising 930 bp upstream of the TSS and 1,892 bp downstream of TSS (comprising of the $5^{\prime}$ untranslated region, first exon, first intron, and second exon, see also Figure S4A) was PCR amplified (Phiuson, Thermo Scientific) and cloned in-frame with GUS ORF into pCAMBIA1301-EcoRI/NcoI (EcoRI-35S promoter-NcoI fragment was previously eliminated from the vector). Cloning was done using the Gibson Assembly method 
(https://sgidna.com). After sequencing the inserted fragments to confirm the absence of mutations caused by PCR, independent transgenic lines were generated in Col-0 via floral dipping using C58C1 agrobacterium strain.

For primers please see Supplementary Materials.

\section{Generation of QK;phsfa1a::HSFA1a-3xHA Plants}

To generate the transgenic line expressing a C-terminally 3xHA-tagged HSFA1a in QK background, the genomic DNA of Arabidopsis (Col-0) HSFA1a comprising the 754 bp sequences upstream of TSS and the full CDS was amplified in a PCR reaction, then fused to a 3xHA coding sequence and the NOS terminator in a binary vector. The construct was then transferred into the Agrobacterium GV3101 strain and transformed into the QK mutant as previously described (Liu and Charng, 2013).

\section{GUS Staining}

Plant material was incubated for $30 \mathrm{~min}$ in $90 \%$ (v/v) acetone on ice, rinsed with $50 \mathrm{mM}$ sodium phosphate buffer, $\mathrm{pH} 7.0$, and incubated overnight at $37^{\circ} \mathrm{C}$ in staining solution $(0.5 \mathrm{mg} /$ $\mathrm{ml} \mathrm{X}$-Gluc [5-bromo-4- chloro-3-indolyl-b-D-glucuronide], $50 \mathrm{mM}$ sodium phosphate buffer, $\mathrm{pH} 7.0,0.5 \mathrm{mM}$ potassium ferrocyanide, $0.5 \mathrm{mM}$ potassium ferricyanide, and $0.1 \%[\mathrm{v} / \mathrm{v}]$ Triton X-100). After staining, samples were washed with $50 \mathrm{mM}$ sodium phosphate buffer, $\mathrm{pH} 7.0$, and cleared in $70 \%$ (v/v) ethanol. The GUS histochemical staining was visualized under a light stereomicroscope (Leica MZ10 F). For each transgenic construct multiple independent lines were assayed (see figure legends).

\section{RNA Extraction and Northern Blotting}

Total RNA was extracted from approximately $30 \mathrm{mg}$ seedlings. The homogenized plant materials were resuspended in $600 \mu \mathrm{l}$ of extraction buffer (0.1 M glycine- $\mathrm{NaOH}, \mathrm{pH} 9.0,100 \mathrm{mM} \mathrm{NaCl}, 10$ mM EDTA, 2\% SDS) and mixed with an equal volume of phenol $\mathrm{pH} 4,3$. The aqueous phase was treated with equal volumes of phenol-chloroform and chloroform, precipitated with ethanol and resuspended in sterile water. RNA gel blot analysis of higher molecular weight RNAs was performed as described previously (Silhavy et al., 2002).

RNA gel blot analysis of 21-24 nt RNAs was performed as follows. Approximately $5 \mu \mathrm{g}$ of total RNA was separated by $15 \%$ polyacrylamide gel electrophoresis (PAGE) with $8.6 \mathrm{M}$ urea and 1x Tris-borate-EDTA. RNA was electroblotted onto Hybond-NX membranes and fixed by chemical crosslinking at $60^{\circ} \mathrm{C}$ for $1 \mathrm{~h}$ (Damm et al., 2015). Small RNA Northern blot hybridization and analysis were performed using complementary DNA oligo for miR824 and miR824-3p or locked nucleic acid oligonucleotides for miR159 and miR398a (Exiqon, http://www.exiqon.com). siRNA Northern blotting assays were done in at least three biological replicates if not otherwise stated.

\section{qRT-PCR}

For qRT-PCR assays, $5 \mu \mathrm{g}$ total RNA was DNase treated according to manufacturer's instructions (Ambion AM2222, www.thermofisher.com), precipitated in ethanol, resuspended in sterile water. One microgram of DNase-treated total RNA and random primer was used for the first-strand complementary DNA reaction according to the manufacturer's instructions (NEB, E6300S, www.neb.com). qPCRs were done using qPCR Master Mix (NEB, M3003S, www.neb.com) according to the manufacturer's instructions. qPCR reactions were run in a Light Cycler 96 (Roche) Real-Time PCR machine. Samples were collected at Zeitgeber Time 8 (ZT8). At least three biological samples were assessed in each experiment and standard error bars shown. P values were calculated using unpaired two-tailed Student t-test to assess the significance of differences. For primers please see Supplementary Materials.

FT mRNA measurements: to avoid derepression of FT by high ambient temperatures, instead of ACCx3 we heat-treated plants by exposing them directly to $37^{\circ} \mathrm{C}$ for $1 \mathrm{~h}$ and cooling back immediately to $21^{\circ} \mathrm{C}$. Treatment was applied repeatedly for 3 days in a row $\left(37^{\circ} \mathrm{C} \times 3\right)$. This dose of moderate HS does not affect the overall growth and survival of the seedlings (Stief et al., 2014). For AGL16 change detection, we collected samples at midday (ZT8), while as FT peaks at the end of the light period we collected samples at the end of the light period (ZT16).

\section{Western Blotting}

Seedlings were homogenized in extraction buffer (150 mM Tris$\mathrm{HCl}, \mathrm{pH} 7.5,6 \mathrm{M}$ urea, 2\% SDS, and 5\% $\mu$-mercaptoethanol). Samples were boiled, and cell debris was removed by centrifugation at $18,000 \times g$ at $4^{\circ} \mathrm{C}$ for $10 \mathrm{~min}$. The supernatants were resolved on $12 \%$ SDS polyacrylamide gel electrophoresis, transferred to Hybond PVDF membranes (GE Healthcare) and subjected to Western blot analysis. For detection 3xHA-tagged HsfAla, horseradish peroxidase conjugated antibody (Roche, 3F10) was used. The proteins were visualized by chemiluminescence (ECL kit; GE Healthcare) according to the manufacturer's instructions.

\section{Chromatin Immunoprecipitation}

ChIP assays were performed using pooled 10-day-old heattreated seedlings. ChIP experiments were done as described (Angel et al., 2011), using the anti-HA Affinity Matrix (Roche, 11815016001). The ChIP data were quantified by qPCR. ACTIN2 was used as an internal reference gene. For primers please see Supplementary Materials.

\section{Physiological Measurements Measurements of Gas Exchange Analyses Under Different Temperature Conditions}

The gas exchange analysis was performed on intact attached leaves of 21-day old plants using a Ciras 3 portable photosynthesis instrument equipped with a narrow $\left(1.7 \mathrm{~cm}^{2}\right)$ leaf cuvette (PP systems, Haverhill MA, United States). For ACCx3 pretreated plants treatments were done on days 18, 19,20. The net assimilation rate $(\mathrm{Pn})$, stomatal conductance (gs), and transpiration rate (E) were determined under two temperature conditions (22 and $37^{\circ} \mathrm{C}$ ) and at steady state of photosynthesis using a $\mathrm{CO}_{2}$ level of $400 \mu \mathrm{l} \mathrm{l}^{-1}$ and light intensity of $700 \mu \mathrm{mol} \mathrm{m} \mathrm{m}^{-2} \mathrm{~s}^{-1}$. 


\section{Temperature-Dependent Chlorophyll a Fluorescence Measurements}

The heat-induced changes of chlorophyll $a$ fluorescence parameters were also detected on intact detached leaves by the use of a pulse amplitude modulated fluorometer (ImagingPAM M series, Walz, Effeltrich, Germany) completed with a thermoregulatory instrument consisting of a water-cooled Peltier thermoelectric module, a thermocouple thermometer, and a control unit. The measurements were started at $21^{\circ} \mathrm{C}$ and after the photosynthesis was steady $(15 \mathrm{~min})$ under actinic light illumination at $100 \mu \mathrm{mol} \mathrm{m}^{-2} \mathrm{~s}^{-1}$ the temperature was increased from 21 to $55^{\circ} \mathrm{C}$ at a rate of $1^{\circ} \mathrm{C} \mathrm{min}^{-1}$. During the measurements, $1.0 \mathrm{~s}$ saturated flashes (photosynthetic photon flux density $=3,000 \mu \mathrm{mol} \mathrm{m}^{-2} \mathrm{~s}^{-1}$ ) provided by an LED-Array Illumination Unit IMAG-MAX/L $(\lambda=450 \mathrm{~nm})$ were applied at each degree Celsius. The effective quantum yield of PS (II) parameter was shown.

\section{In situ Hybridization}

In situ hybridization was done as described before (Medzihradszky et al., 2014). For probe preparation, AGL16 or PIN1 complementary DNA was cloned into the pBSK+ vector. RNA probe was prepared using T7 in vitro transcription, based on manufacturer's instructions (Thermo). As AGL16 antisense had a high background and agl16-1 negative control could not be used (since contains AGL16-T-DNA transcript) we used PIN1 antisense RNA as a negative control (Figure 5B). PIN1 mRNA is not expressed in leaf veins but is strongly expressed in shoot apical meristem.

\section{Large Datasets: RNA Transcriptome Analysis}

Total RNA samples of 7 days old Col-0 and agl16-1 seedlings have been prepared for Illumina sequencing (in four biological replicates each). Paired-end library preparation was done using TruSeq Stranded mRNA LT Sample Prep Kit according to TruSeq Stranded mRNA Sample Preparation Guide (Part \# 15031047 Rev. E). Raw RNAseq data have been made available in the SRA repository (SRP151884). RNA reads were aligned to the Arabidopsis genome (TAIR10) (Lamesch et al., 2012) using hisat2-2.1.0 (Kim et al., 2015). Bedgraph files have been generated using SAMtools 1.8 (Li et al., 2009) and deepTools2 (Ramirez et al., 2016) and visualized by Integrated Genome Browser (v9.0.0) strand specifically (Freese et al., 2016).

\section{RESULTS}

miRNAs are 10 times more stable than mRNAs on average, having a lifespan of several days (Csorba et al., 2010; Gantier et al., 2011; Sanei and Chen, 2015). We hypothesized that due to their high stability, the stress-responsive miRNAs might gradually accumulate in response to recurring stress cues and act as lasting memory factors to fine-tune their targets on longer terms after stress. To find miRNAs responsive to heat and potentially involved in HS adaptation, we treated plants using an HS program that mimics natural conditions (Mittler et al., 2012; Ling et al., 2018) (Figure 1A). Gradual treatments were applied by elevating temperature from 21 to $37^{\circ} \mathrm{C}$ in the course of four hours for three days in a row (acclimation treatment, ACCx3) (see also Materials and Methods). To understand how stable the changes are caused by ACCx 3 treatment, we monitored the plants 1 day after acclimation (recovery sample, REC). We also subjected another batch of plants to HS 1 day after acclimation (ACCx3 + HS).

\section{Recurring Heat Stress Causes Accumulation of miR824 and Stable Decrease of AGL16 mRNA Level}

By applying heat acclimation repeatedly, among others, we have found miR824-5p and miRNA star strand miR824-3p to accumulate (Figures 1B, C). Stepwise accumulation of miR824-5p could be clearly observed when plants were exposed to an increasing number of ACC treatments (Figures 1B, C). miR824-5p and $-3 p$ were maintained at high levels after treatment (Figures 1B, C, recovery samples), (hereafter miR824-5p will be referred to as miR824).

To find out whether miR824 accumulation is due to transcriptional induction, we checked the level of its precursors. All spliced forms, including pri-miR824 and pre-miR824, were found at elevated levels in the ACCx3 but dropped to the background the next day (Figure 1B, middle panel). The fast turnover of precursors is most likely due to the quick splicing and dicing processes. Higher levels of unspliced-miR824 RNA were observed when plants were exposed to $45^{\circ} \mathrm{C}$ following acclimation (ACCx3 + HS, Figure 1B). This is likely caused by the stress-induced transcription coupled with inefficient splicing of nascent transcripts known to occur during HS (Ling et al., 2018). miR824, $-3 p$ or precursors did not accumulate in plants grown at elevated ambient temperatures $\left(7\right.$ days at $27^{\circ} \mathrm{C}$, Figure 1B). This finding was also confirmed by studying small RNA (sRNA) deep sequencing data published before (Gyula et al., 2018). miR824 transcriptional induction, therefore, is a bona fide stress response.

To confirm that miR824 is induced transcriptionally by heat, we created $\beta$-glucuronidase (GUS) reporter lines driven by the miR824 promoter ( $p 824_{W T}: G U S$ ) (Figures 1D, E and Figure S1). As a control, we used $p 35 S:: G U S$ lines. The GUS activity was detected in the vasculature of leaves, stomata guard cells and trichomes as reported earlier (Hu et al., 2014). Besides these, GUS staining was observed in the actively dividing tissues including the root apical meristem, calyptra, veins and branching points, the shoot apical meristem, the flowering stem branching points, the tip of the siliques, and the veins of the floral organs (Figure S1A). We analyzed the HS-responsiveness of the miR824 promoter by monitoring GUS mRNA (Figure 1E and Figure S1B): GUS mRNA was strongly elevated at $37^{\circ} \mathrm{C}$ and slightly increased at $45^{\circ} \mathrm{C}$ (Figure 1E). GUS transcription driven from $\mathrm{p} 35 \mathrm{~S}$ promoter (p35S::GUS) was very mildly affected (Figure 1E and Figure S1B). These findings suggest that recurring, moderately high temperature $\left(37^{\circ} \mathrm{C}\right)$ spells lead to the gradual accumulation of 
$\mathbf{A}$

(i)

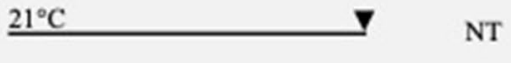

(ii)<smiles>CCCCCCCCCCCCCC(C)(C)C</smiles>

(iii)

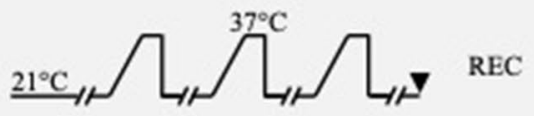

(iv)

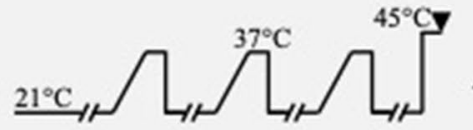

$\mathrm{ACCx} 3+\mathrm{HS}$

(v)

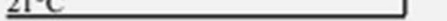

HS

B
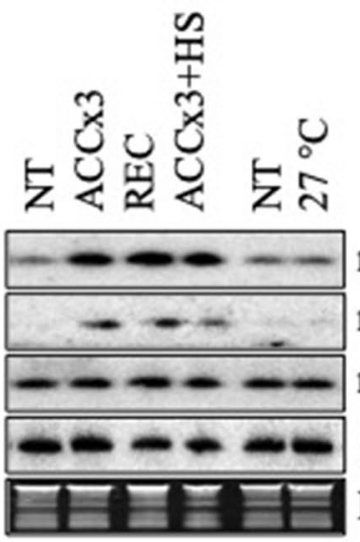

miR824-5p

miR824-3p

miR159

U6

$\mathrm{EtBr}$

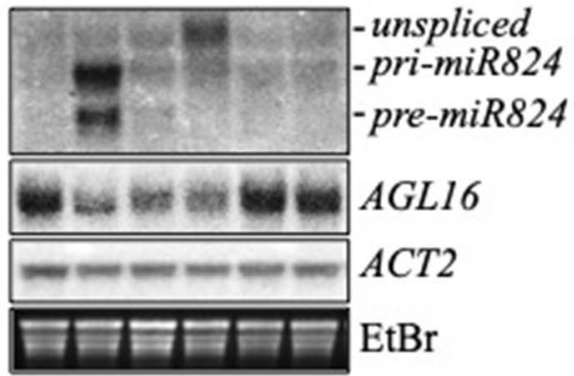

D

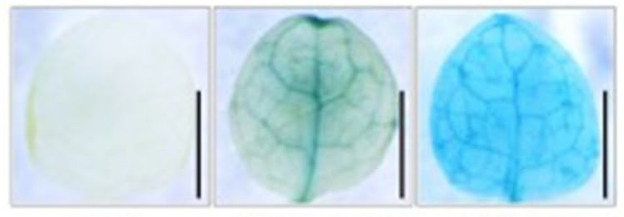

Col-0 p824 $\because$ :GUS p35S::GUS

$\mathbf{E}$

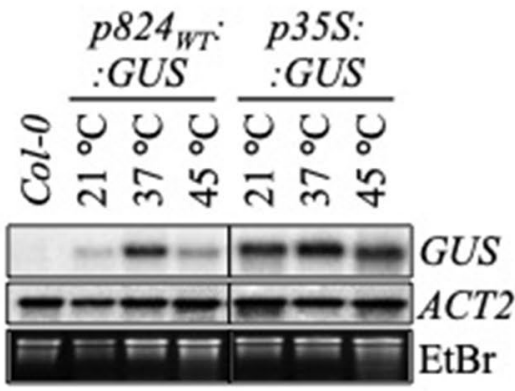

FIGURE 1 | Recurring gradient acclimation causes the accumulation of miR824 and stable depletion of AGL16 messenger RNA (mRNA). (A) Heat stress regimes used in the study. (i) nontreated, NT; (ii) acclimation repeated three times, ACCx3: heat gradient elevated in the course of 4 h; (iii) 1 day recovery following ACCx3 treatment, REC; (iv) heat stress following acclimation, ACC $\times 3+\mathrm{HS}$; (v) direct heat stress, HS; temperatures are shown on top of each regime, timeline is shown on bottom, samples were all collected at midday; (B) AGL16, miR824, and miR824-precursor changes in response to the treatments depicted in (A); miR824-5p and $-3 p$ accumulates following repeated gradient acclimation and remains high during a day recovery. miR824 precursor forms (unspliced, pri-miR824, and pre-mir824) are transiently induced by heat but not $27^{\circ} \mathrm{C}$. High temperatures cause a persistent decrease in AGL16; treatments are shown on the top; (C) miR824 gradually accumulates in response to recurring heat: acclimation repeated once, twice or three times (ACC, ACCX2, or ACC 3 ); 1 day recovery, (+1d REC) (quantification of Northern blot data; bars represent standard errors based on three biological replicates; p values based on two-tailed Student's $t$-test, NT value was set to 1.); (D) GUS staining of the first rosette leaf of 2-week-old Col-0 control plant, and plants transformed with p824 wT::GUS or p35S::GUS control reporter constructs. (E) miR824 promoter-driven GUS transcription is induced by heat treatment in p824 wT::GUS transgenic plants; Col-0 and p35S::GUS plants were used as controls; ACTIN2 (ACT2) and ethidium-bromide staining (EtBr) are shown as loading controls. 
mature miR824 through the combination of repeated transient transcriptional activation and stability of the miRNA after stress. miR824, therefore, may integrate transient or sporadic HS signals that are reflected in its cumulated levels (Figure 1C).

Parallel to the miR824 accumulation, the miR824 target AGL16 mRNA was stably depleted (Figure 1B). To understand how the heat-induced AGL16 downregulation relates to the AGL16 levels in the AGL16 mutant (agl16-1) or the miR824 mutant plants, we analyzed Col-0, agl16-1 (SALK_104701), $\triangle 824$ (SALK_138988), and a previously characterized miR824mimicry MIM824 (Hu et al., 2014) lines during NT and ACC treatments (Figure 2). Downregulation of the AGL16 mRNA under elevated temperatures was very efficient as it reached similar levels compared to the agl16-1 mutant (Figure 2A). Heat-induced AGL16 changes were confirmed by quantitative real-time PCR (qRT-PCR) as well (Figure 2C).

A clear signal was detected in the agl16-1 plants by Northern blot (Figure 2A). The T-DNA insertion in agl16-1 is within the last exon that may give rise to a truncated transcript. Besides qRT-PCR analysis (Figure 2C) we confirmed this by genotyping (Figure S2A) and RNAseq analysis (Figure S2B); (for the remnant signal of AGL16mRNA detected by Northern blot in agl16-1 plants please see Supplementary Information). The primiR824 induction and miR824 accumulation were not affected by the agl16-1 mutation (Figures 2A, B).

We analyzed miR824 and AGL16 mRNA levels in the miR824 mutants ( $\triangle 824$ and MIM824) as well. In the $\Delta 824$ mutant the T-DNA insertion disrupts the MIR824 gene (located within the pri-miR824 but not the pre-miR824 transcript region). Induction of the miR824 transcription was detected during heat treatment (unspliced), but the pri- and pre-miR824 maturation was largely impaired (Figure 2A). In spite of this, a residual amount of mature miR824 accumulates, and the level of AGL16 mRNA is moderately increased in the $\Delta 824$ plants (nonsignificant vs. Col-0, Figures 2A, C). The residual amount of miR824 in the $\triangle 824$ plants, therefore, is enough to limit AGL16 mRNA levels

\section{A}
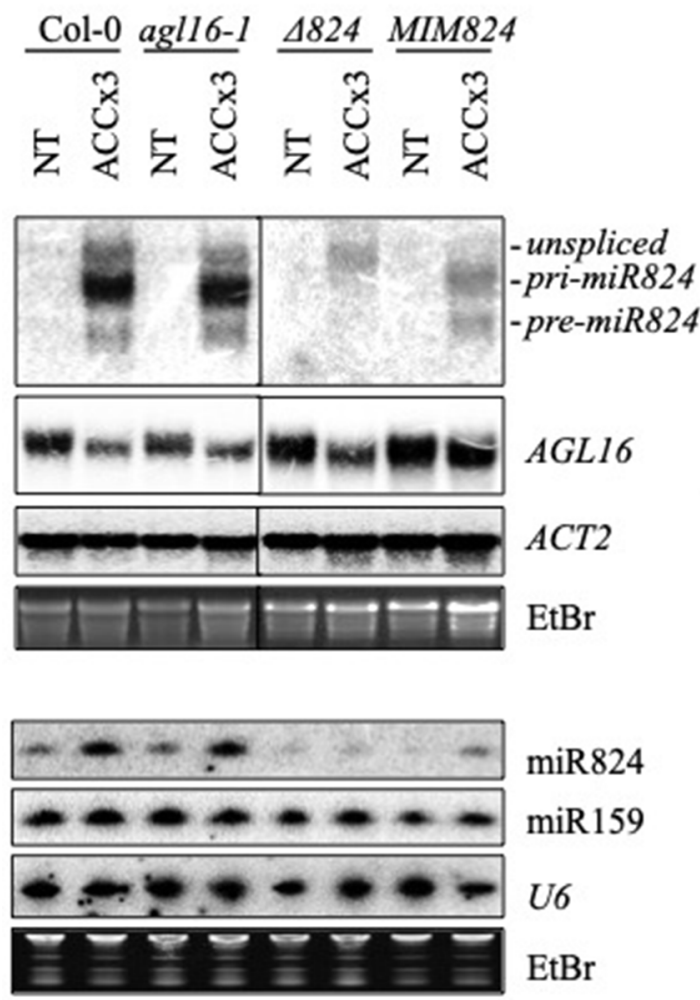

$\operatorname{miR} 824$

$\operatorname{miR} 159$

U6

$\mathrm{EtBr}$
B

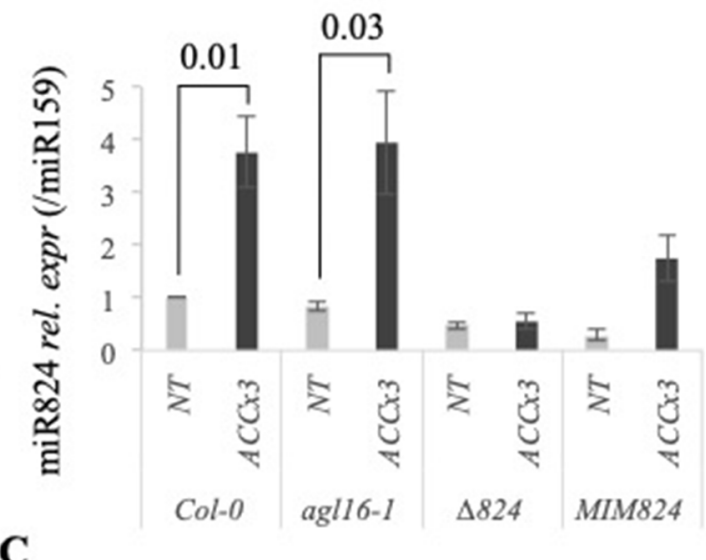

$\mathbf{C}$

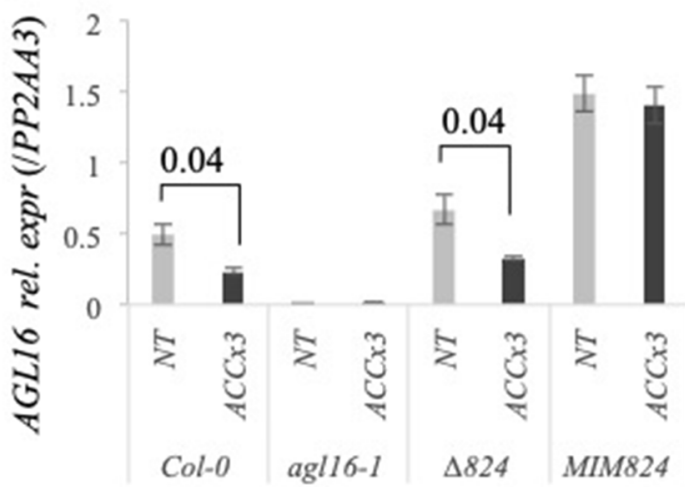

FIGURE 2 | Heat-induced changes of miR824 precursors, miR824, and AGL16 messenger RNA (mRNA) in the different miR824/AGL16 module mutants. (A) Northern blot assays showing miR824-precursors, miR824 and AGL16 mRNA levels in Col-0 wild type, ag/16-1, miR824-mutant ( $\Delta 824)$, or miR824 mimicry (MIM824) plants; (nontreated, NT; acclimated three times, ACCx3); miR159, U6, ACTIN2, and ethidium-bromide (EtBr) staining are shown as loading controls.

(B) Quantification of miR824 Northern blot signals in the different mutant backgrounds; bars represent standard errors based on three biological replicates; Col-0 NT value was set to 1. (C) Quantitative real-time PCR quantification of AGL16 mRNA relative expression in the different mutants; bars represent standard errors based on three biological replicates; $p$ values based on two-tailed Student's $t$-test. 
very efficiently. Heat-induced AGL16 mRNA reduction was not altered in the $\Delta 824$ plants suggesting the involvement of a miR824-independent mechanism (Figures 2A, C).

In the MIM824 line, a much stronger increase of AGL16 mRNA could be observed (three-fold, Figures 2A, C) in agreement with earlier data ( $\mathrm{Hu}$ et al., 2014). The MIR824 transcriptional induction and the mature miR824 accumulation were decreased in the MIM824 plants. Although the mature miR824 accumulates (Figures 2A, B), its activity is efficiently neutralized by the presence of the target mimicry transcript RNA (Franco-Zorrilla et al., 2007; Hu et al., 2014) that leads to strong accumulation of AGL16. In MIM824 the impact of heat on the expression of AGL16 was limited suggesting a miR824dependent and miR824-independent complex regulation (Figure 2C).

As agl16-1 and MIM824 plants were both shown to have a clear physiological phenotype (Kutter et al., 2007; Hu et al., 2014), heat stress induction of miR824 and downregulation of AGL16 may play a role in HS adaptation.

\section{A Dual Mechanism for AGL16 Downregulation During Heat Stress}

As miR824 was proved to regulate AGL16 directly and negatively (Kutter et al., 2007; Hu et al., 2014), and because the expression of miR824 and AGL16 anticorrelated during HS a direct causality was suspected. Strikingly, we observed a decrease of AGL16 expression during early $\mathrm{HS}$, when the induction of miR824 was not yet detectable (direct $45^{\circ} \mathrm{C}$ for $30 \mathrm{~min}$, Figure S3A). This suggests that the decrease of $A G L 16$ expression at high temperatures is miR824-independent. This is also corroborated by the AGL16 dynamics in the miR824-mutants ( $\triangle 824$ and MIM824) during HS (Figure 2C) and suggests a complex mechanism.

To separate the miR824-dependent and miR824-independent downregulation of AGL16 during HS, we analyzed its mRNA levels in the miR824-defective plants. We compared AGL16 downregulation and recovery during 4 days following a single ACC treatment in Col-0, 4824 , and MIM824 plants (Figure 3). A single ACC treatment was applied to achieve a transient induction, so we could monitor the half-life and the lasting activity of the mature miR824. The time scale was chosen because the sRNA-loaded RISCs were shown to be stable and active for several days (Csorba et al., 2010). The pri-miR824 transcription and the miR824 accumulation were efficiently induced by the single ACC treatment in Col-0 plants, to a lower extent in MIM824 plants but not in $\Delta 824$ mutant (Figures $3 \mathrm{~A}-\mathrm{E}$ ). The AGL16mRNA levels immediately dropped in all genotypes suggesting a miR824-independent mechanism (Figure 3F). In the recovery period, the $A G L 16$ downregulation was maintained only in the Col-0 plants (Figures 3A, F); in the $\Delta 824$ mutant (where only a low level of miR824 is present) or MIM824 plants (where miR824-RISC is inactive), the AGL16 level was quickly restored by the next day (Figures 3B, C, and F). These findings show that the AGL16 downregulation is caused by a miR824dependent and a miR824-independent mechanism. On the other hand, these findings also suggest, that the miR824-dependent pathways' effect could be two-sided: it may contribute to the immediate downregulation of its target and can serve to keep it repressed following the acute stress period.

Next, we aimed to understand the miR824-independent decrease of AGL16. The mRNA abundance depends both on the rate of RNA transcription and RNA decay. To unpick these routes, we assayed AGL16 unspliced mRNA (uAGL16) levels as a proxy for transcriptional changes (Figure S3B). The uAGL16 mRNA level was slightly (nonsignificantly) lower in response to heat treatment indicating that AGL16 transcription may be affected. To assess the AGL16 promoter activity by a second approach, we created pAGL16::GUS reporter lines (Figure S4A) and assayed GUS mRNA transcription under NT and ACC conditions (Figure S4B). The AGL16 promoter activity was decreased in all lines. GUS transcript abundance was mildly induced by $p 35 S$ promoter ( $p 35 S:: G U S$ was used as a control). These results suggest that the miR824-independent decrease of AGL16, at least in part, could be due to a transcriptional downregulation.

Next, we considered the possibility of an elevated decay of AGL16 mRNA during HS. Cytoplasmic SKI-exosome $3^{\prime}-5^{\prime}$ exonuclease complex may contribute to the AGL16 degradation during HS (van Hoof et al., 2002; Halbach et al., 2013). We analyzed the changes of AGL16 expression in the ski2-2 mutant (Figure S3C). RISC 5' cleavage fragment of AGL16 was partially stabilized in ski2-2 as was shown in the case of other miRNA targets (Branscheid et al., 2015; Szadeczky-Kardoss et al., 2018). The RISC 5' cleavage fragment stabilization occurred under both NT and HS conditions. These findings suggest that miR824loaded RISC can operate at elevated temperatures and that the SKI-exosome complex may clear RISC 5' fragments under both NT and HS. Full-length AGL16 mRNA decrease was not impaired in ski2-2 compared to Col-0.

XRN4 is the main cytoplasmic $5^{\prime}-3^{\prime}$ RNA exonuclease (Souret et al., 2004; Gy et al., 2007; Gregory et al., 2008). XRN4, together with its cofactor LARP1 was shown to play a role in the degradation of unneeded RNA species during early HS (Merret et al., 2013; Merret et al., 2015). We analyzed AGL16 mRNA dynamics under heat treatment in xrn4-6 (Gy et al., 2007) and found similar downregulation of full-length AGL16 as in Col-0 control (Figure S3C). Based on these, we failed to attribute any role of SKI-exosome complex or XRN4 in the HS-mediated miR824-independent full-length AGL16 mRNA decay.

\section{HS-Induction of miR824 Transcription Requires HSE cis-Element}

To better understand the heat-mediated transcriptional regulation of miR824, we studied its promoter in silico. We predicted a heat shock element $[\operatorname{HSE}(1)]$ at -925 to -915 upstream from the transcriptional start site (TSS) and further two corrupted HSE-like motifs [HSE-like (2) and HSE-like (3)] at -796 to -785 and -661 to -651 upstream from the TSS, respectively (Figure 4A). To verify whether these motifs are functional, we employed promoter mutation analysis of our GUS reporter (Figure 4B and Figure S1). The functionality of the HSE elements was assessed by introducing point mutations to generate a single mutant $\left(p 824_{H S E 1}:: G U S\right)$ and a triple mutant $\left(p 824_{\text {HSE123 }}: G U S\right)$ promoter-driven GUS reporter line. Basal 
$\mathbf{A}$

々 $\frac{\text { REC }}{+1+2+3+4} \circlearrowright \frac{\text { REC }}{<+1+2+3+4}$

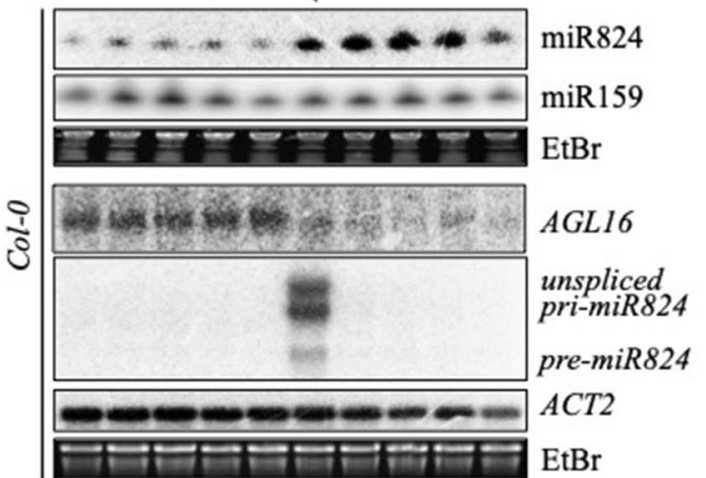

$\mathbf{B}$

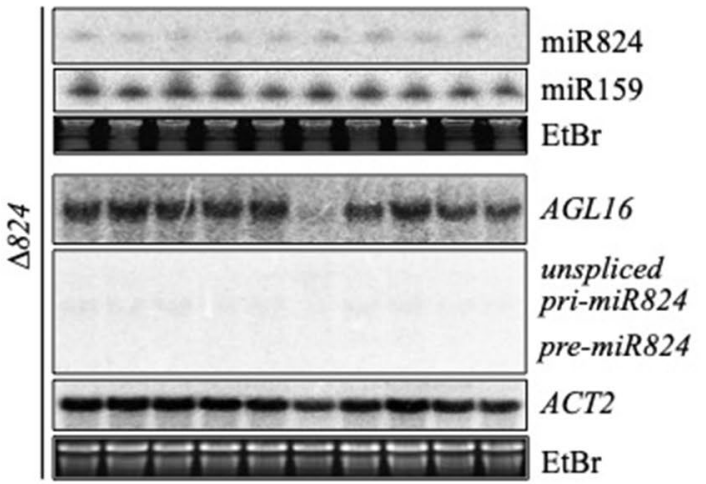

D

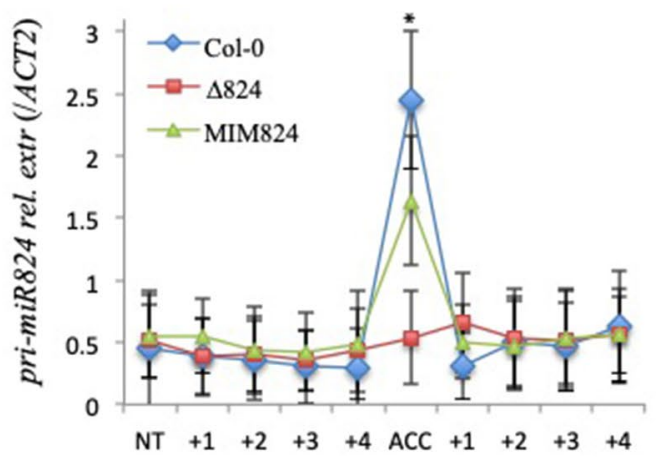

$\mathbf{E}$

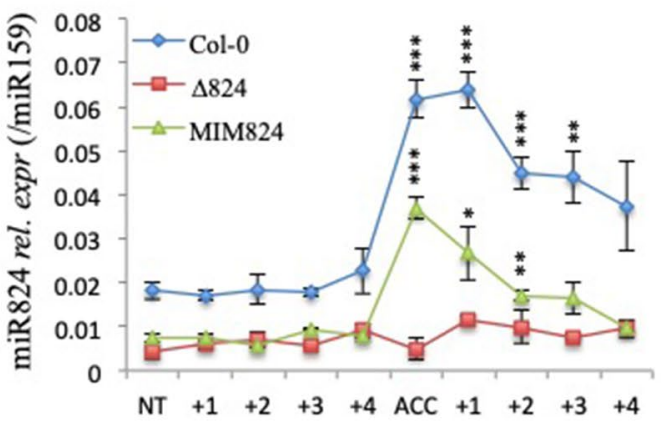

$\mathbf{F}$

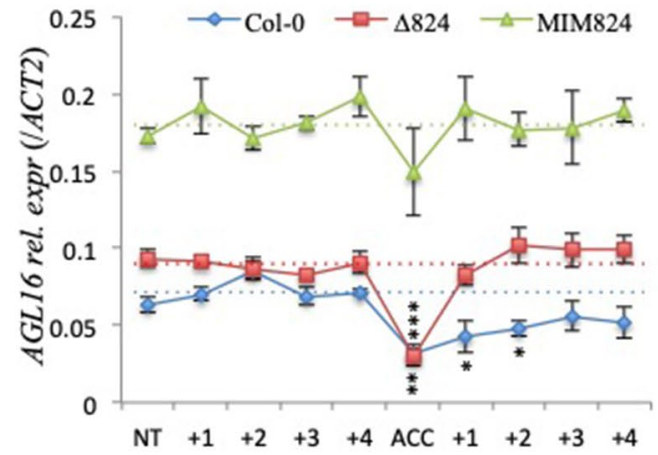

FIGURE 3 | Dual depletion of AGL16 messenger RNA (mRNA) during and following heat stress (HS). (A-C) Levels of miR824-precursor, mature miR824, and AGL16 RNA changes during a time-course following a single treatment (ACC) or nontreated control (NT) (treatments are shown on the top: $+1,+2,+3,+4$ denote days passed after the treatment); (A) Col-0 plant samples, (B) miR824-mutant $\Delta 824$ plant samples and (C) miR824-mimicry MIM824 plant samples; miR159, ACTIN2, and EtBr staining are shown as loading controls; (D-F) Quantification of at least three sets of Northern blot measurements: (D) pri-miR824 data, (E) mature miR824 data, and (F) AGL16 mRNA data; quantification data were first quantified to miR159 or ACTIN2 controls than to nontreated controls; dotted lines represent basal level of AGL16 in the wild-type and miR824-mutants; bars represent standard errors of three biological replicates; $p$ values based on two-tailed Student's $t$-test $\left({ }^{\star} p<0.05,{ }^{\star *} p<0.01,{ }^{\star \star *} p<0.001\right)$.

and ACC-induced GUS mRNA expression of multiple lines was analyzed by Northern blotting and the signals were quantified (Figure 4B and Figure S1B). Heat induction of the promoter was abolished already when the single HSE element was mutated (Figure 4A and Figure S1). The expression levels driven from the $p 824_{H S E 1}$ and $p 824_{\text {HSE } 123}$ promoters were very similar. These results suggest that the HSE motif at -925 to -915 upstream from TSS is functional.

Besides the HSE cis-elements, we also found a predicted MADS-box binding site in the promoter of MIR824 (at -805 to 
$\mathbf{A}$

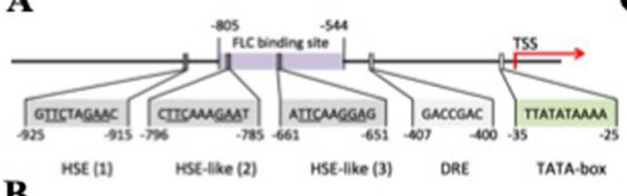

$\mathbf{B}$

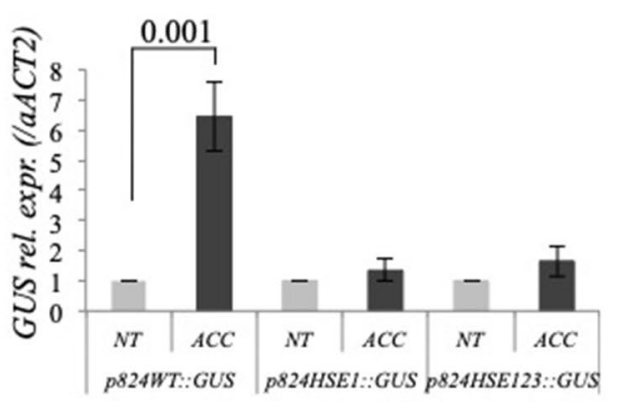

D

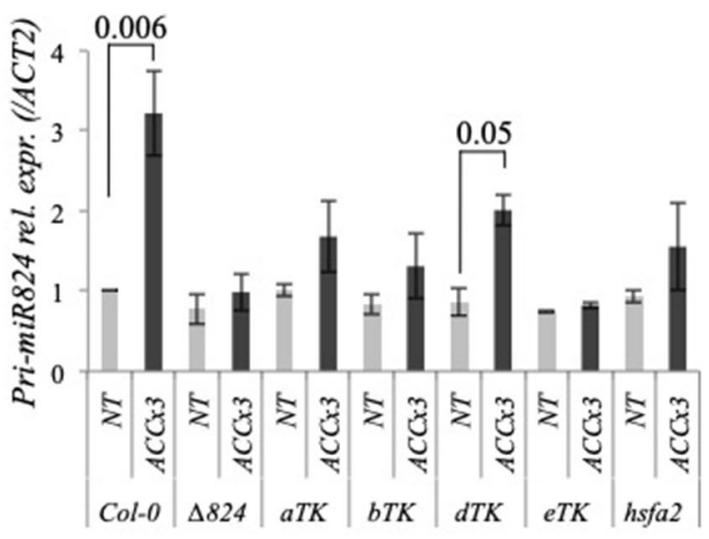

C
Col-0 4824 aTK $\quad b T K \frac{d T K}{e T K} \underline{h s f a 2}$
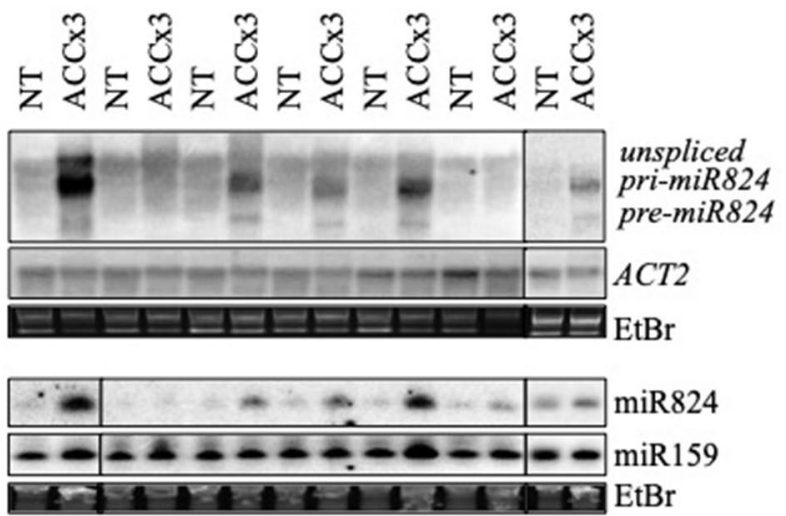

$\mathbf{E}$

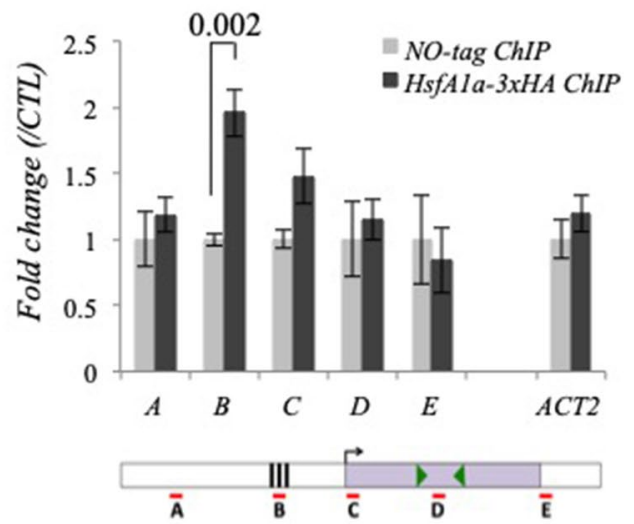

FIGURE 4 | miR824 transcriptional induction requires heat stress elements and heat shock factors. (A) Schematic representation of $M I R 824$ gene promoter: heat stress element, HSE; heat stress element-like motifs, HSE-like; dehydration-responsive element, DRE; TATA-box, FLC-binding site (based on Deng et al., 2011), arrow shows transcription start site, TSS; nucleotide base numbers are relative to TSS. (B) Northern blot data of GUS expression using the wild-type

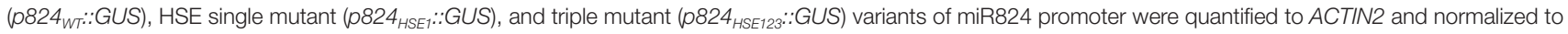
nontreated control; nontreated, NT, acclimated, ACC; bars represent standard errors calculated based on six representative independent lines each. (C) miR824precursor induction and miR824 accumulation are faulty or partial in miR824-mutant $\Delta 824$, triple mutants of HSFA1 family (aTK, bTK, dTK, and eTK) and HSFA2 mutant hsfa2. miR159, ACTIN2, and ethidium-bromide (EtBr) staining are shown as loading controls. (D) Quantification of pri-miR824 signals of Northern blots; bars represent standard errors based on three biological replicates; Col-0 NT value was set to 1. (E) chromatin immunoprecipitation (ChIP) qRT-PCR using 3xHA-tagged HsfA1a expressing transgenic plants: HsfA1a binds to the HSE-containing region of miR824 promoter; data were normalized to no-tag ChIP control; bars represent standard errors calculated based on two biological and two technical reps, schematic representation of MIR824 locus is shown below: $A$, B, C, D, E segments show the locations of PCR amplicons; black boxes show the location of HSE elements; green arrowheads show locations of miR824-5p and miR824-3p; $p$ values based on two-tailed Student's t-test.

-544 from TSS, Figure 4A) using plantdhs.org web tool (Zhang et al., 2016). This motif was confirmed as an FLC binding site based on the FLC ChIPseq data of Deng and coworkers (Deng et al., 2011). The presence of the FLC binding motif suggested a possible feedback regulation through an FLC-AGL16 interaction (Hu et al., 2014). To assess the biological relevance of the motif, we checked the miR824 expression in the FLC mutant $f l c-2$, wildtype Col-0, and FLC overexpressing Col-FRI plants (Michaels and Amasino, 1999) (Figure S5). No differences of pri-miR824 levels could be found between the genotypes under NT or ACC conditions. We cannot exclude however, that FLC with or without AGL16 protein may regulate miR824 expression to finetune AGL16 mRNA levels under more erratic conditions.

\section{miR824 HS-Induction Requires HSFA1 Family Members and HSFA2 trans-Factors}

The functional HSE element present in the promoter and transcriptional induction of miR824 during elevated temperatures suggested that heat shock factors (HSFs) might be involved in the process. To determine which HSF is required for pri-miR824 transcription, we analyzed the miR824 expression in several HSF 
mutants (Figures 4C, D and Figure S6A). In the triple knockout mutants expressing only one family member of the HSFA1 family [aTK, bTK, and $d T K$, (Liu et al., 2011)] the induction of pri-miR824 was partial while in the triple hsfala; hsfalb; hsfald (eTK) mutant it was completely lost (Figures 4B, C). The miR824 HS-induction was partially abolished also in the $h s f a 2$ mutant (Figures 4C, D) but not in the $h s f a 3, h s f a 6 a, h s f a 6 b, h s f a 7 a$, and $h s f a 7 b$ mutants (Figure S6A). The requirement of HSFA1s and HSFA2 factors for heatinduced miR824 transcription was also confirmed by the decreased accumulation of the mature miR824 in the respective mutant backgrounds (Figure 4C). The basal expression of pri-miR824 or AGL16 was not significantly altered in the hsfala; hsfalb; hsfald; hsfale (QK) (Liu et al., 2011) or in the hsfa2 mutant (Figures S6B, C).

To unravel whether HSFA1s are required for miR824 heatinduction directly or indirectly, we generated transgenic lines expressing 3xHA-tagged HsfAla from its own promoter in a $Q K$ background (QK;pHsfA1a::HSFA1a-3xHA). The 3xHA-tagged HSFA1a was shown to fully complement the HS phenotype of the $Q K$ plants (Figure S7A) and to be efficiently expressed under HS (Figure S7B). We subjected this line to ACC treatment and performed chromatin immunoprecipitation assay (Figures S7C, D) followed by real-time quantitative PCR analysis (ChIP-qPCR) (Figure 4E). We have found that the genomic DNA located in the HSE-containing promoter region but not the upstream or downstream regions of the MIR824 locus or ACTIN2 locus are enriched by HSFA1a-3xHA-ChIP relative to the control sample (Figure 4E). These findings are consistent with HSFA1a directly regulating miR824 promoter to activate transcription during heat.

\section{The MIR824 Locus Does Not Possess Transcriptional Memory}

HS memory-related genes show a faster or stronger transcriptional response upon recurring $\mathrm{HS}$ in a process that required the activity of HSFA2 (Lamke et al., 2016). The observation that miR824 transcriptional induction involves HSFA2 activity prompted us to analyze transcriptional memory of the MIR824 locus. For this, we treated plants repeatedly for one, two or three times (ACC, ACCx2, ACCx3) (Figure S8). High and similar levels of pri-miR824 were detected regardless of the number of acclimations (Figure S8A). miR824 induction was neither faster nor stronger, even though a high level of HSFAla protein was available following the first acclimation (Figure S8B). Upon ceasing of heat treatment the pri-miR824 RNA signal dropped back to background showing that miR824 transcription is neither sustained poststress (Figure 3D and Figure S8A). We reasoned that the extended ACC program ( $4 \mathrm{~h}$ in total) may saturate the transcriptional induction of MIR824 gene locus and therefore we may miss the early events. As enhanced transcriptional activation of genes with active transcriptional memory is already apparent after $15 \mathrm{~min}$ in response to recurring HS (Liu et al., 2018), we repeated the experiment by applying short treatments $\left(37^{\circ} \mathrm{C} / 15 \mathrm{~min}\right.$ each). Transcriptional induction of pri-miR824 was very quick and of similar amplitude regardless of the number of treatments (Figure S8C). Based on these we conclude that MIR824 locus does not possess transcriptional memory.

\section{The miR824/AGL16 Module Is Not Directly Involved in Heat Stress Response}

miR824/AGL16 module was reported to be a regulator of stomata development (Kutter et al., 2007; Yang et al., 2014). Water evaporation through stomata cools the surface of the leaves preventing HS damage. To unravel if AGL16 downregulation during and following HS has an impact on thermotolerance of photosynthetic apparatus through stomata complexity regulation we measured stomata conductance (gs), $\mathrm{CO}_{2}$ assimilation (Pn), transpiration (E) rates, and thermotolerance of the photosynthetic apparatus PS II in NT and ACCx3 plants (Col-0, agl16-1, 4824 , and MIM824) at both 25 and $37^{\circ} \mathrm{C}$ (Figures S9-S11). No significant changes or consistent trends could be observed between the different genotypes indicating that the temperature-dependent changes of the photosynthetic apparatus are not related to the miR824/AGL16 module (for more details see Supplementary Information). In accordance with these, we could not find differences in growth and survival rates of AGL16 or miR824 mutants following basal thermotolerance and short acquired thermotolerance assays [based on (Charng et al., 2007)].

\section{AGL16 Tissue-Specific Expression Overlaps with miR824 and FT}

The other described function of AGL16 is the regulation of flowering transition under light-dark conditions through FT pathway. Manipulation of miR824 abundance in the MIM824 line contributed to the modulation of FT levels and flowering time change (Kobayashi and Weigel, 2007; Hu et al., 2014). Previously it was shown that expression of FT and GUS activity (expressed from pro-miR824::GUS transgene) localizations are very similar, namely in leaf vasculature (Kobayashi and Weigel, 2007; Hu et al., 2014). However, the tissue-specific localization of AGL16 was not studied. We analyzed the tissue-specific promoter activity of the AGL16 in transgenic plants expressing GUS transgene from AGL16 promoter (using pAGL16::GUS construct) (Figure 5A, $i$-iii, and Figure S4C). GUS activity was detected in seedling and rosette leaf veins, root calyptra, root cell division, elongation zone, and vasculature, trichomes, shoot apical meristem. We confirmed vasculature localization of AGL16 mRNA by in-situ hybridization (Figure 5A, iv-vi and Figure S4D). The vascular-specific expression of AGL16 in the leaves overlaps with the expression of both miR824 (Figure 1D and Figure S1A) and FT (Takada and Goto, 2003). These findings extend earlier observations (Burgeff et al., 2002; Hu et al., 2014) and further underpin the role of the miR824/AGL16 module in FT regulation.

\section{AGL16 Downregulation During and Following Heat Stress May Fine-Tune FT Levels}

The impact of the miR824/AGL16 module on FT and flowering acceleration was more pronounced in the background that expressed FLC at high levels (e.g. FRIGIDA) (Hu et al., 2014). We crossed our mutants into the Col-FRI background (ColFRI/agl16-1, Col-FRI/D824, and Col-FRI/MIM824) and tested 
$\mathbf{A}$

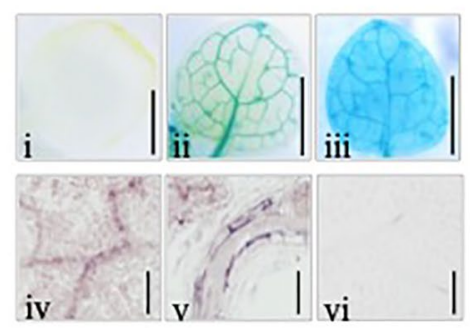

B

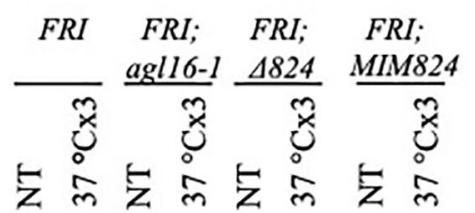

C

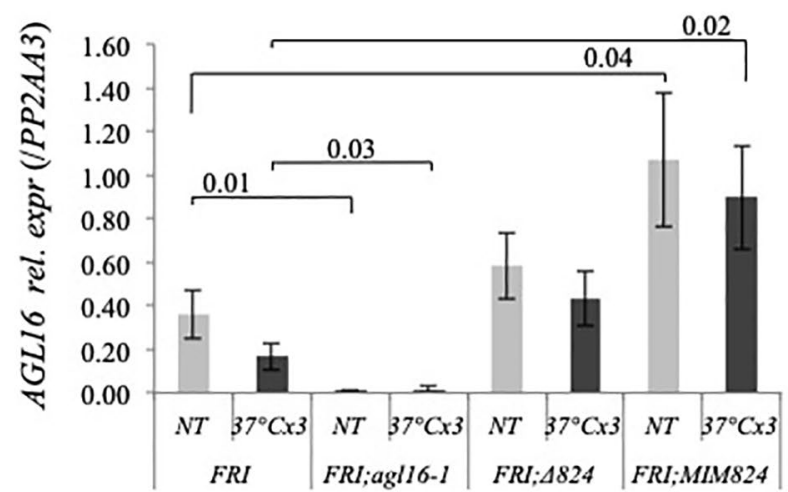

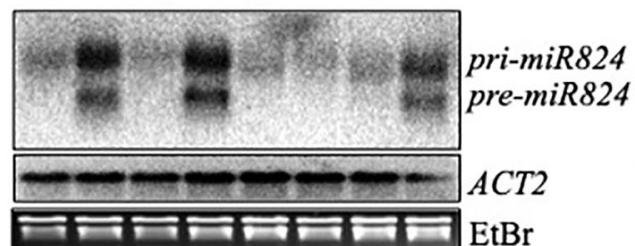

D
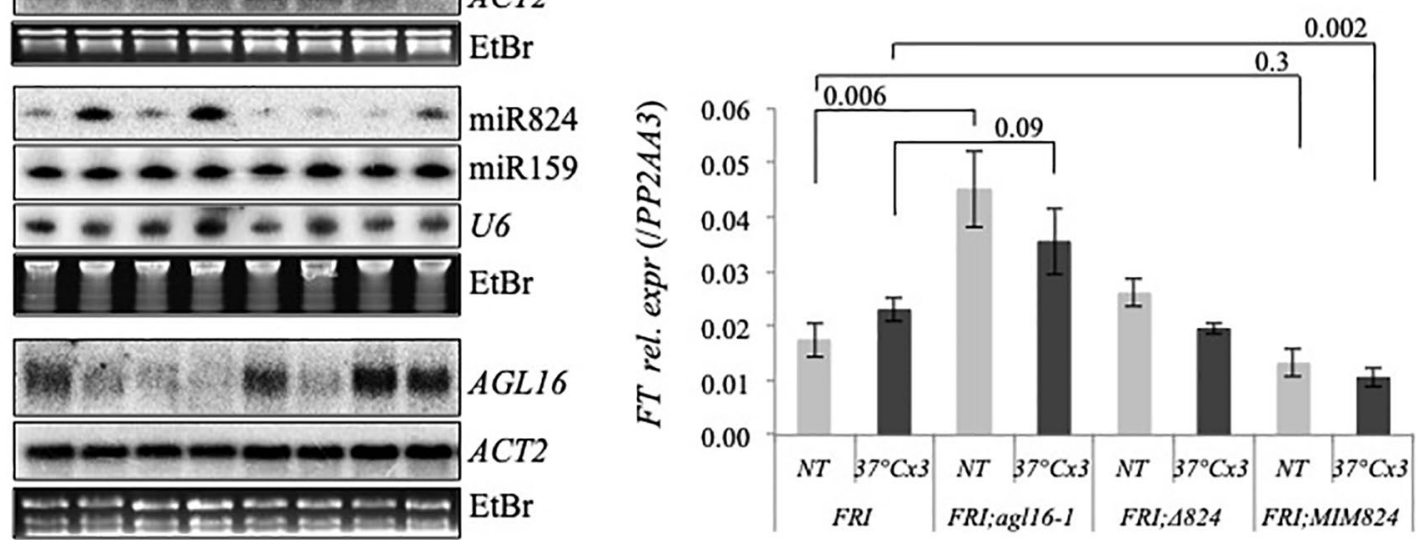

FIGURE 5 | Tissue-specific expression of AGL16 and expression changes in winter accession Arabidopsis. (A) AGL16 expression is specific to vasculature: $\beta$-glucuronidase (GUS) activity staining of (i) nontransformed control; (ii) pAGL16::GUS transformed; and (iii) p35S:::GUS transformed seedling leaf (10 day old true leaf, gray bar: $1 \mathrm{~mm}$ ); AGL16 messenger RNA (mRNA) in situ hybridization of 10 days old seedling: (iv) leaf, (v) root, and (vi) PIN1 mRNA hybridization control in leaf (gray bar: $0.1 \mathrm{~mm}$ ); (B) miR824-precursor, miR824, and AGL16 mRNA level changes in Col-FRI wild-type and mutant plant (samples are shown on the top); ACTIN2, miR159, U6, and ethidium-bromide (EtBr) staining are shown as loading controls. (C) Heat-induced relative expression changes of AGL16 in Col-FRI wildtype and mutants; bars represent standard errors based on three biological replicates; $p$ values based on two-tailed Student's $t$-test. (D) $F T$ changes in response to repeated mild HS in wild-type and mutant plants; bars represent standard errors based on six biological replicates; $p$ values based on two-tailed Student's $t$-test.

the impact of HS on miR824, AGL16, and FT (Figures 5B, C). To avoid the impact of elevated ambient temperature on FT (McClung et al., 2016), instead of gradient heat treatments we employed direct $37^{\circ} \mathrm{C}$ repeatedly (see Materials and Methods). This heat treatment enabled efficient induction and accumulation of miR824, and downregulation of AGL16; the changes recapitulated the ones found in Col-0 background (Figures 2A, C). miR824/AGL16 module heat-regulation, therefore, occurs in both summer (Col-0) and winter (Col-FRI) ecotypes of Arabidopsis.

To unravel the impact of AGL16 repression on FT, we analyzed its mRNA changes in wild type (Col-FRI) and mutant
(Col-FRI;agl16-1, Col-FRI;A824, and Col-FRI;MIM824) plants (Figure 5D). In Col-FRI;agl16-1 the FT mRNA level was significantly elevated (NT Col-FRI;agl16-1 vs. NT Col-FRI, $\mathrm{p}=0.006$ ) with a 2.6 -fold difference, similarly as shown before (Hu et al., 2014). Following heat treatment, this difference was partially lost (1.5-fold difference, nonsignificant). In ColFRI; $\triangle 824$ the FT mRNA levels were not significantly different from those in Col-FRI (in both NT and heat-treated samples) (Figures 5B, C). Although only mild changes of AGL16 are detected in this mutant background the slightly higher FT levels (in NT samples) contradicted the expectations (the reason for this is unknown at the moment). In NT Col-FRI;MIM824 the 
FT mRNA levels were lower compared to NT Col-FRI (0.75-fold difference, nonsignificant). This is in agreement with the finding that AGL16 levels are high in the absence of miR824 activity (Figure 5C). FT levels dropped significantly following heat treatment in Col-FRI;MIM824 (0.46-fold difference, $\mathrm{p}=0.002$, Figure 5D). As FT changes were mild we wanted to corroborate these findings: we analyzed FT changes using another internal control (UBC22 mRNA) and got similar results (Figure S12).

These results suggest that HS has a complex impact on FT, probably through multiple pathways, including AGL16independent and AGL16-dependent ones. The heat-induced downregulation of AGL16 (in wild-type) may cause a mild derepression of FT. In the absence of AGL16 changes (e.g. ColFRI;agl16-1 or miR824 mutants), the level of FT slightly drops, suggesting that HS may impact it negatively through AGL16independent pathways. miR824/AGL16 module, therefore, may compensate for the retarding impact of heat under mild HS conditions (in wild type), while in agl16-1 and miR824 mutants, where this buffer system is not available, the negative impact of HS on FT becomes apparent (see also Discussion). Prompted by the observation on FT changes, we tried to assay the impact of HS on the timing of the flowering transition. Unfortunately, we could not detect consistent differences in flowering time following our heat treatments between mutants and wild type plants (see also Discussion).

\section{miR824/AGL16 HS-Regulation Is Conserved Within Brassicaceae}

Both miR824 and AGL16 (containing miR824-RISC target site) are conserved within Brassicaceae (Kutter et al., 2007; de Meaux et al., 2008). We performed a complementary experiment to check whether HS-regulation of miR824/AGL16 functional module is conserved. First, we assayed miR824 behavior in response to ACCx3 in multiple members of Brassicaceae ( $B$. rapa, Brassica oleracea, Brassica napus, and R. sativus). miR824 accumulated in all Brassica plants tested (Figure 6A). To confirm that miR824 accumulation is due to transcriptional induction, we checked miR824 precursors in B. napus winter variety Darmor (containing active FRI paralogs) and the summer variety RV31 (Westar derivative) by qRT-PCR: pri-miR824 was elevated following heat acclimation in both varieties (Figure 6B). In parallel to this, AGL16 mRNA downregulation also occurred in the two B. napus varieties (Figure 6C). Altogether these observations suggest that HS-regulation of miR824/AGL16 module is conserved within Brassicaceae, and may have a role in fine-tuning adaptation following mild and repeated HS.

\section{DISCUSSION}

Plants respond to diurnal and seasonal changes in temperature by reprogramming their developmental pathways. It is known that the impact of HS largely depends on the strength, type, and duration of stress or the developmental stage of the plants when stress is encountered (Yeh et al., 2012). While the effect of direct HS on miRNA regulation has been intensively studied
(Ballen-Taborda et al., 2013; Guan et al., 2013; Cui et al., 2014; Kruszka et al., 2014; Stief et al., 2014; Liu et al., 2015) much less is known about how gradual and repeated high temperatures affect miRNAs. Our HS program was designed to mimic natural conditions involving both the gradual and repeated aspects. Regimes involving gradual stress buildup are physiologically relevant since they resemble natural situations. Recurring high or low temperatures, gradual onset of drought and salt may all lead to the accumulation of stress-responsive miRNAs.

Using this program we have found that miR824 is a HS responsive miRNA. We have characterized in detail the changes of the miR824/AGL16 module in response to heat and in the poststress period. Using an in silico analysis and GUS reporter assay we demonstrated that MIR824 gene promoter contains a functional HSE cis element. We also propose that HSFAla trans factor may directly bind to the HSE containing region of MIR824 promoter (Figure 4E). Besides HSE, we have also found a drought-responsive element in the promoter of MIR824 (GACCGAC, -407 to -400 from TSS) (Figure 4A). The combination of heat and drought induced strong transcription of pri-miR824 and accumulation of miR824 (Figure S13). Beyond these, miR824 was shown to be downregulated by arsenic stress in B. juncea (Srinivasan et al., 2006) and to accumulate under chromium stress in $R$. sativus L. (Liu et al., 2015). On the other hand, AGL16 homolog genes in B. rapa, BraMADS20 and BraMADS21 showed differential accumulation in response to cold or salt stress (Saha et al., 2015). Our findings and data from the literature suggest that miR824/AGL16 pathway may integrate the stimuli of multiple abiotic stresses under complex climatic conditions. It remains a future task to understand the role of miR824/AGL16 module under combined stresses.

We show that although miR824 transcription is transient, mature miR824 accumulates gradually to high levels following repeated heat treatments (Figure 1C). Similarly, stress-induced changes of miR168 and miR171a-1 were found by using repeated HS regime $\left(42^{\circ} \mathrm{C} / 3 \mathrm{~h}\right.$ per day/7 days in a row) (Bilichak et al., 2015; Liu et al., 2015). As miRNAs possess long half-life (Csorba et al., 2010; Gantier et al., 2011; Sanei and Chen, 2015) they may be capable to act as integrators of stress signals over a few days. The exploitation of heat spikes through miR824/AGL16 module changes may serve plants for monitoring the seasonal progression, similarly as shown before in another system (Hepworth et al., 2018).

We studied miR824 unique target AGL16 and have shown that its downregulation in response to high temperatures is dual, comprising of a miR824-independent and a miR824-dependent route (see Working Model, Figure 7). AGL16 nascent transcript level and promoter activity are decreased miR824-independently during heat (Figures S3B and S4B), therefore transcriptional ceasing contributes to AGL16 downregulation. As reduction is abrupt, mRNA decay may be also involved (Figure S3C). HS-mediated decay was shown to be an important component of HSR (Merret et al., 2013; Merret et al., 2015). To confirm our findings, we have analyzed the transcriptome changes in larp1 RNAseq data (Merret et al., 2013) and xrn4-5 RNAseq data (Merret et al., 2015): AGL16 transcript was enriched in neither larp1 nor xrn4-5 vs. wild-type as it would be expected if XRN4-LARP1 
$\mathbf{A}$

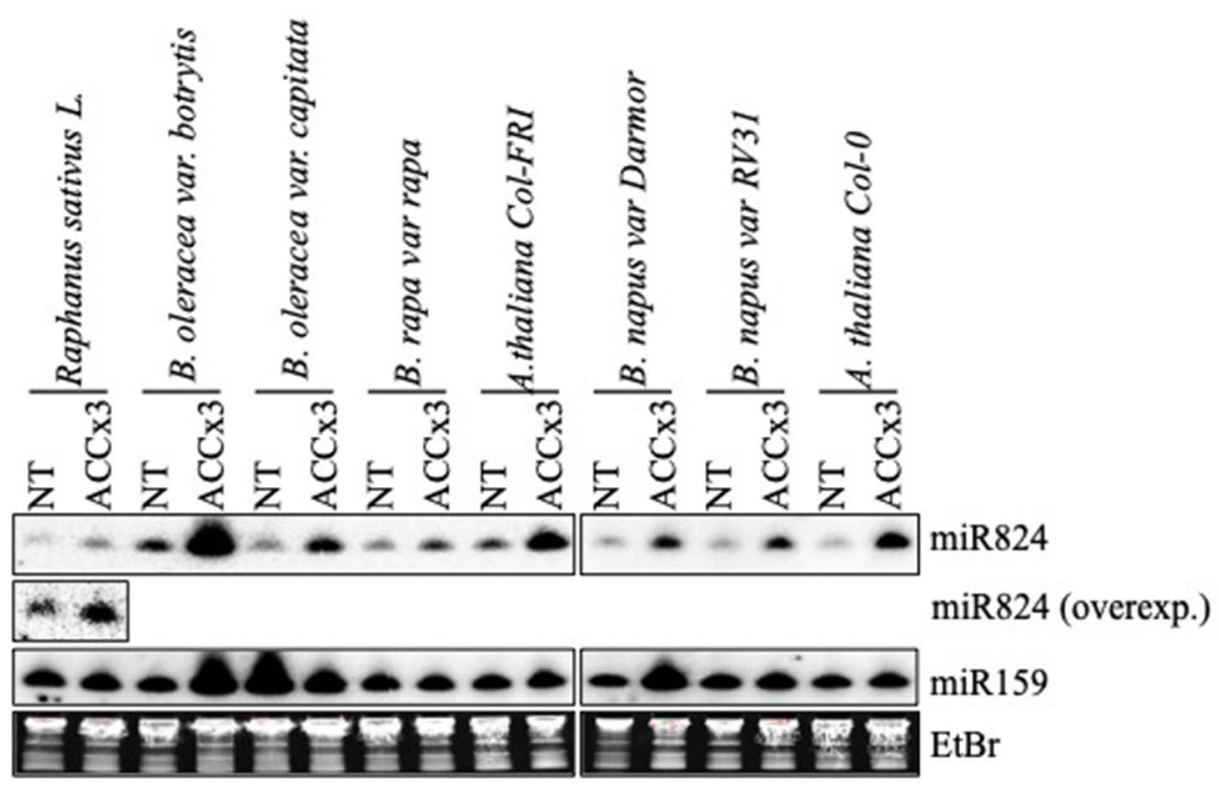

B
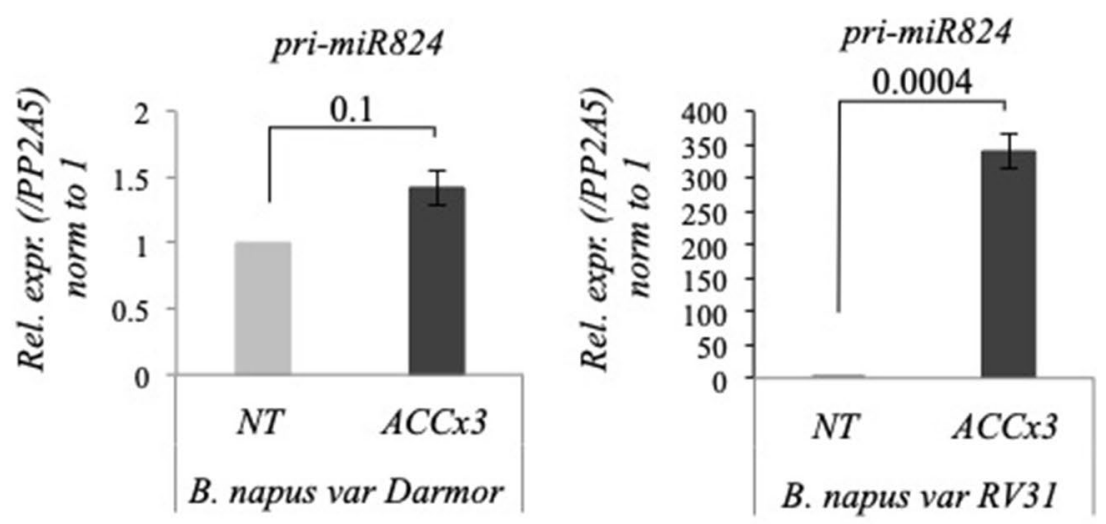

C

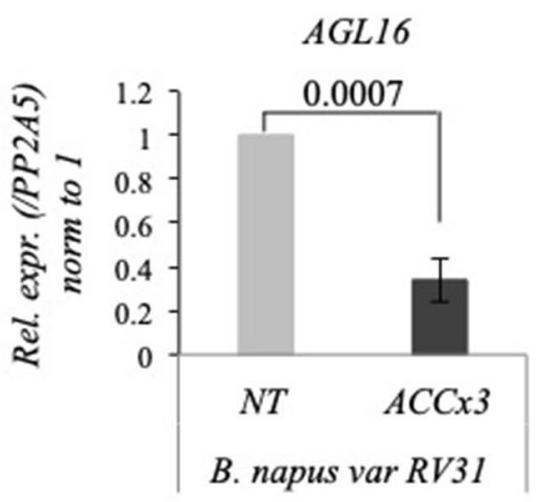

FIGURE 6 | Heat-regulation of miR824 and AGL16 is conserved in Brassica species. (A) miR824 accumulates in Brassicaceae family members in response to recurring acclimation. miR159 and ethidium-bromide (EtBr) staining are shown as loading controls. (B) pri-miR824 changes in B. napus winter cultivar Darmor and the spring cultivar RV31. (C) BnaAGL16 is significantly depleted during repeated acclimation in B. napus; NT value was set to 1. bars represent standard errors based on three biological replicates; $p$ values based on two-tailed Student's $t$-test. 


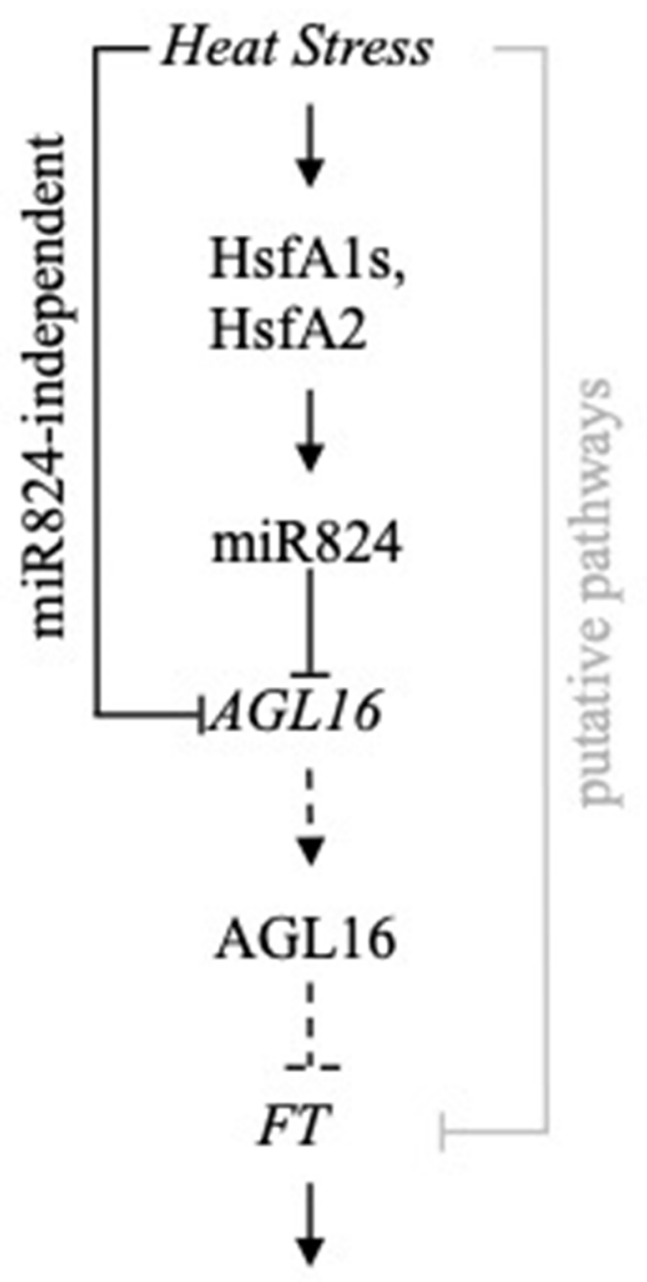

Flowering transition

FIGURE 7 | Proposed working model of miR824/AGL16 module heat stress regulation. Heat stress induces transcription of miR824 through HSFA1a family and HSFA2 transcription factors. AGL16 is depleted through a miR824-dependent and a miR824-independent pathway. Stable downregulation of $A G L 16$ leads to derepression of $F T$, a central integrator of flowering transition (dotted lines depict downregulated steps during and following HS). FT level may be also altered by other putative heat stress (HS)-regulated factors (gray line).

pathway is involved in its degradation. HS-induced AGL16 transcript changes found by (Merret et al., 2015) are remarkably similar to the ones found by us: AGL16 is downregulated to 0.62 in wild-type Col-0 and 0.69 in $x r n 4-5$ following $30 \mathrm{~min}$ of HS. It is possible that $3^{\prime}-5^{\prime} \mathrm{XRN} 4$ and $5^{\prime}-3^{\prime} \mathrm{SKI}$-exosome pathway act redundantly to contribute to AGL16 mRNA decay. miRNAindependent heat-induced downregulation of miRNA targets was observed in other cases as well: ARF10, 16 and 17 targets of miR160 were partially and significantly downregulated by heat even when the effect of miR160 was blocked through the expression of mimicry constructs (Lin et al., 2018).

Several lines of evidence support the involvement of miR824dependent pathway in downregulation of AGL16: (i) basal levels of AGL16 in wild-type (both Col-0 and Col-FRI) is lower compared to the miR824-pathway deficient $\Delta 824$ and MIM824 lines; consistent with this, AGL16 level is elevated in dcl1-8, hyl11, and hen1-1 mutants (Kutter et al., 2007; Confraria et al., 2013) (ii) repression of AGL16 following heat treatment persists for several days only in wild-type plants but not miR824-deficient mutants; (iii) RISC 5' cleavage fragments in ski2-2 plants become stabilized in both NT and HS samples. This latter observation also suggests that RNA silencing is active during HS.

Importantly, we show that the extended presence of miR824loaded RISC prolongs the effect of heat and maintains the low levels of AGL16 target poststress (Figure 3). miR156 family members are also at elevated level poststress and this results in stable downregulation of target SQUAMOSA-PROMOTER BINDING-LIKE (SPL) transcription factors mRNA (Stief et al., 2014). As suggested by the authors, the lasting high level of miR156 mature form is probably due to the combination of transcriptional memory of the locus and the high miRNA stability (Stief et al., 2014). In the case of miR824 we excluded transcriptional memory of the locus (Figure S8). Transient HS-inducibility (absence of sustained transcription poststress) of miR824, therefore, provided us an excellent tool to measure its half-life and track its downstream effects (instead of using general transcription inhibitors like cordycepin that have a strong pleiotropic impact). Following a single heat treatment, during which the miR824 transcription is transiently switched on, we have shown that the mature miRNA persists and is active for several days (Figure 3). In addition to these, we analyzed the heatinducible miR398a (Guan et al., 2013) and found elevated levels of it 3-4 days poststress (Figure S14). As stress-induced miRNAs are stably present poststress, as shown for miR824, miR398a (in this study) or miR156, miR831 (Stief et al., 2014), and active in repressing their targets, we propose they should be regarded as posttranscriptional stress-memory factors. The lasting effect of stress-induced miRNAs enables plants to "remember" the recent occurrence of stress and helps to alter the poststress development on a few days timescale or during intermittent periods between stresses. Whether stress-induced sRNAs act as memory factors during other or combined stress conditions remain a future and exciting question.

The timing of flowering is a critical trait that ensures the perfect timing of seed production required for species survival. Transition to flowering is regulated by an elaborate network involving numerous players based on endogenous and exogenous stimuli. Age, circadian clock, sugar content, temperature, and hormonal pathways converge on a limited number of master regulators (Romera-Branchat et al., 2014; Whittaker and Dean, 2017). Temperature is one of the most important environmental stimuli to modulate transition timing from vegetative to reproductive phase. It is known that elevated ambient temperature accelerates flowering time in Arabidopsis (Balasubramanian and Weigel, 2006; Capovilla et al., 2015; McClung et al., 2016). How nonlethal HS affects flowering time is much less understood. 
Our data suggest that AGL16 downregulation during and following HS may contribute to a mild derepression of FT. In wild-type plants, FT is slightly elevated, while in AGL16/miR824 mutants is rather repressed. We hypothesize that the AGL16/ miR824 module may serve as a buffer system to dampen the effects of HS that retards the flowering transition (Figure 7). Unfortunately, we could not detect flowering time changes following our HS program. Several factors could hinder this. As the heat treatment is mild and plants are exposed for a relatively short period, our treatment may have a limited impact/ potential to cause lasting changes that culminate in flowering. Proper timing of HS is also critical and difficult to predict. Furthermore, FT-independent pathways (Wang et al., 2009) also alter flowering time. One such pathway studied in detail is the miR156/SPL module pathway (Stief et al., 2014). SPLs are master regulators of developmental transitions and accelerate flowering in an FT-independent manner (Wang et al., 2009). HS-induced miR156 negatively regulates SPLs (Stief et al., 2014). miR156/ SPL pathway coordinates the balance between development and stress response in the favor of the latter, to delay flowering (Cui et al., 2014; Stief et al., 2014) therefore act in the opposite direction (compared to the impact of miR824/AGL16). Recently it was shown that Arabidopsis plants exposed to prolonged mild HS temperatures $\left(30^{\circ} \mathrm{C}\right.$ for 7 days) bolted earlier and that early flowering phenotype was transmitted trans-generationally for two generations (Liu et al., 2019). Early flowering and transgenerational thermomemory were caused by a complex regulatory network that culminates in the release of HEATINDUCED TAS1 TARGET 5. HEAT-INDUCED TAS1 TARGET 5 drove early flowering in a process involving FT upregulation (Liu et al., 2019). In summary, better understanding the interaction between positive and negative regulators and combined impact on developmental transitions following nonlethal HS conditions remains a future and exciting challenge.

FT and FLC orthologs are key genes that contribute to flowering time control and a successful adaptation to diverse environmental conditions and geographical distribution in the different B. napus ecotypes (Wu et al., 2019). We show that HS regulation of the miR824/AGL16 module is conserved in several Brassica species and characteristics to both summer and winter varieties (Figure 6). The paralog $r s a$-miR824/AGL16 module in radish was also related to bolting and flowering processes (Nie et al., 2015). HS regulation of the miR824/AGL16 module may,

\section{REFERENCES}

Alvarez-Buylla, E. R., Liljegren, S. J., Pelaz, S., Gold, S. E., Burgeff, C., Ditta, G. S., et al. (2000). MADS-box gene evolution beyond flowers: expression in pollen, endosperm, guard cells, roots and trichomes. Plant J. 24 (4), 457466. doi: 10.1111/j.1365-313X.2000.00891.x

Angel, A., Song, J., Dean, C., and Howard, M. (2011). A Polycomb-based switch underlying quantitative epigenetic memory. Nature 476 (7358), 105-108. doi: 10.1038 /nature 10241

Arora, R., Agarwal, P., Ray, S., Singh, A. K., Singh, V. P., Tyagi, A. K., et al. (2007). MADS-box gene family in rice: genome-wide identification, organization and expression profiling during reproductive development and stress. BMC Genomics 8, 242. doi: 10.1186/1471-2164-8-242 therefore, help successful adaptation of Brassica species and finetune the trade-off between stress and development.

\section{DATA AVAILABILITY STATEMENT}

Raw RNAseq data have been made available in the SRA repository (SRP151884).

\section{AUTHOR CONTRIBUTIONS}

TC conceived the original research plans and designed the experiments. HMS and TC performed most of the experiments and analyzed the data. ÉD and TJ performed physiological measurements. H-CL and Y-YC prepared the phsfala::HsfA1$3 x H A$-tagged transgenic plant lines and provided technical assistance. AM performed the in situ hybridizations. TC wrote the article with contributions of all the authors.

\section{FUNDING}

TC was supported by the János Bolyai Research Scholarship of Hungarian Academy of Science. Y-YC was supported by a grant from the Ministry of Science and Technology, Taiwan (103-2311-B-001-011-MY3). Hungarian Scientific Research Fund OTKA K115934, K129283 funded this work.

\section{ACKNOWLEDGMENTS}

We thanks to J. de Meaux for providing us the MIM824 line, to H. Vaucheret for xrn4-6 mutant, C. Dean for $f l c-2$, and JA. Irwin for B. napus seeds. We are grateful to Szittya lab and D. Silhavy for their help on bioinformatics analysis and comments on the manuscript. HMS is a Ph.D. student at the Eötvös Lóránd University, Budapest.

\section{SUPPLEMENTARY MATERIAL}

The Supplementary Material for this article can be found online at: https://www.frontiersin.org/articles/10.3389/fpls.2019.01454/ full\#supplementary-material

Axtell, M. J. (2013). Classification and comparison of small RNAs from plants. Annu. Rev. Plant Biol. 64, 137-159. doi: 10.1146/annurev-arplant-050312-120043

Balasubramanian, S., and Weigel, D. (2006). Temperature Induced Flowering in Arabidopsis thaliana. Plant Signal Behav. 1 (5), 227-228. doi: 10.4161/psb.1.5.3452

Ballen-Taborda, C., Plata, G., Ayling, S., Rodriguez-Zapata, F., Becerra LopezLavalle, L. A., Duitama, J., et al. (2013). Identification of Cassava MicroRNAs under Abiotic Stress. Int. J. Genomics 2013, 857986. doi: 10.1155/2013/857986

Barciszewska-Pacak, M., Milanowska, K., Knop, K., Bielewicz, D., Nuc, P., Plewka, P., et al. (2015). Arabidopsis microRNA expression regulation in a wide range of abiotic stress responses. Front. Plant Sci. 6, 410. doi: 10.3389/fpls.2015.00410

Becker, A., and Theissen, G. (2003). The major clades of MADS-box genes and their role in the development and evolution of flowering plants. Mol. Phylogenet. Evol. 29 (3), 464-489. doi: 10.1016/S1055-7903(03)00207-0 
Bilichak, A., Ilnytskyy, Y., Woycicki, R., Kepeshchuk, N., Fogen, D., and Kovalchuk, I. (2015). The elucidation of stress memory inheritance in Brassica rapa plants. Front. Plant Sci. 6, 5. doi: 10.3389/fpls.2015.00005

Borges, F., and Martienssen, R. A. (2015). The expanding world of small RNAs in plants. Nat. Rev. Mol. Cell Biol. 16 (12), 727-741. doi: 10.1038/nrm4085

Branscheid, A., Marchais, A., Schott, G., Lange, H., Gagliardi, D., Andersen, S. U., et al. (2015). SKI2 mediates degradation of RISC 5'-cleavage fragments and prevents secondary siRNA production from miRNA targets in Arabidopsis. Nucleic Acids Res. 43 (22), 10975-10988. doi: 10.1093/nar/gkv1014

Brodersen, P., Sakvarelidze-Achard, L., Bruun-Rasmussen, M., Dunoyer, P., Yamamoto, Y. Y., Sieburth, L., et al. (2008). Widespread translational inhibition by plant miRNAs and siRNAs. Science 320 (5880), 1185-1190. doi: 10.1126/ science. 1159151

Brzezinka, K., Altmann, S., and Baurle, I. (2018). BRUSHY1/TONSOKU/ MGOUN3 is required for heat stress memory. Plant Cell Environ. doi: 10.1111/ pce. 13365

Brzezinka, K., Altmann, S., Czesnick, H., Nicolas, P., Gorka, M., Benke, E., et al. (2016). Arabidopsis FORGETTER1 mediates stress-induced chromatin memory through nucleosome remodeling. Elife 5, pii, e17061. doi: 10.7554/eLife.17061

Burgeff, C., Liljegren, S. J., Tapia-Lopez, R., Yanofsky, M. F., and Alvarez-Buylla, E. R. (2002). MADS-box gene expression in lateral primordia, meristems and differentiated tissues of Arabidopsis thaliana roots. Planta 214 (3), 365-372. doi: 10.1007/s004250100637

Capovilla, G., Schmid, M., and Pose, D. (2015). Control of flowering by ambient temperature. J. Exp. Bot. 66 (1), 59-69. doi: 10.1093/jxb/eru416

Charng, Y. Y., Liu, H. C., Liu, N. Y., Chi, W. T., Wang, C. N., Chang, S. H., et al. (2007). A heat-inducible transcription factor, HsfA2, is required for extension of acquired thermotolerance in Arabidopsis. Plant Physiol. 143 (1), 251-262. doi: 10.1104/pp.106.091322

Charng, Y. Y., Liu, H. C., Liu, N. Y., Hsu, F. C., and Ko, S. S. (2006). Arabidopsis Hsa32, a novel heat shock protein, is essential for acquired thermotolerance during long recovery after acclimation. Plant Physiol. 140 (4), 1297-1305. doi: 10.1104/pp.105.074898

Chen, C., Begcy, K., Liu, K., Folsom, J. J., Wang, Z., Zhang, C., et al. (2016). Heat stress yields a unique MADS box transcription factor in determining seed size and thermal sensitivity. Plant Physiol. 171 (1), 606-622. doi: 10.1104/ pp.15.01992

Chen, L., Ren, Y., Zhang, Y., Xu, J., Sun, F., Zhang, Z., et al. (2012). Genome-wide identification and expression analysis of heat-responsive and novel microRNAs in Populus tomentosa. Gene 504 (2), 160-165. doi: 10.1016/j.gene.2012.05.034

Chen, X. (2004). A microRNA as a translational repressor of APETALA2 in Arabidopsis flower development. Science 303 (5666), 2022-2025. doi: 10.1126/ science. 1088060

Choi, K., Kim, J., Hwang, H. J., Kim, S., Park, C., Kim, S. Y., et al. (2011). The FRIGIDA complex activates transcription of FLC, a strong flowering repressor in Arabidopsis, by recruiting chromatin modification factors. Plant Cell 23 (1), 289-303. doi: $10.1105 /$ tpc. 110.075911

Clough, S. J., and Bent, A. F. (1998). Floral dip: a simplified method for Agrobacterium-mediated transformation of Arabidopsis thaliana. Plant J. 16 (6), 735-743. doi: 10.1046/j.1365-313x.1998.00343.x

Confraria, A., Martinho, C., Elias, A., Rubio-Somoza, I., and Baena-Gonzalez, E. (2013). miRNAs mediate SnRK1-dependent energy signaling in Arabidopsis. Front. Plant Sci. 4, 197. doi: 10.3389/fpls.2013.00197

Csorba, T., Lozsa, R., Hutvagner, G., and Burgyan, J. (2010). Polerovirus protein P0 prevents the assembly of small RNA-containing RISC complexes and leads to degradation of ARGONAUTE1. Plant J. 62 (3), 463-472. doi: 10.1111/j.1365-313X.2010.04163.x

Csorba, T., Questa, J. I., Sun, Q., and Dean, C. (2014). Antisense COOLAIR mediates the coordinated switching of chromatin states at FLC during vernalization. Proc. Natl. Acad. Sci. U. S. A. 111 (45), 16160-16165. doi: 10.1073/pnas.1419030111

Cui, L. G., Shan, J. X., Shi, M., Gao, J. P., and Lin, H. X. (2014). The miR156-SPL9DFR pathway coordinates the relationship between development and abiotic stress tolerance in plants. Plant J. 80 (6), 1108-1117. doi: 10.1111/tpj.12712

Damm, K., Bach, S., Muller, K.M., Klug, G., Burenina, O.Y., Kubareva, E.A., et al. (2015). Improved Northern blot detection of small RNAs using EDC crosslinking and DNA/LNA probes. Methods Mol. Biol. 1296, 41-51. doi: 10.1007/978-1-4939-2547-6_5
De Bodt, S., Maere, S., and Van de Peer, Y. (2005). Genome duplication and the origin of angiosperms. Trends Ecol. Evol. 20 (11), 591-597. doi: 10.1016/j. tree.2005.07.008

de Meaux, J., Hu, J. Y., Tartler, U., and Goebel, U. (2008). Structurally different alleles of the ath-MIR824 microRNA precursor are maintained at high frequency in Arabidopsis thaliana. Proc. Natl. Acad. Sci. U.S.A. 105 (26), 89948999. doi: 10.1073/pnas. 0803218105

Deng, W., Ying, H., Helliwell, C. A., Taylor, J. M., Peacock, W. J., and Dennis, E. S. (2011). FLOWERING LOCUS C (FLC) regulates development pathways throughout the life cycle of Arabidopsis. Proc. Natl. Acad. Sci. U. S. A. 108 (16), 6680-6685. doi: 10.1073/pnas.1103175108

Fernandez, D. E., Wang, C. T., Zheng, Y., Adamczyk, B. J., Singhal, R., Hall, P. K., et al. (2014). The MADS-Domain Factors AGAMOUS-LIKE15 and AGAMOUSLIKE18, along with SHORT VEGETATIVE PHASE and AGAMOUS-LIKE24, Are Necessary to Block Floral Gene Expression during the Vegetative Phase. Plant Physiol. 165 (4), 1591-1603. doi: 10.1104/pp.114.242990

Ferrandiz, C., Gu, Q., Martienssen, R., and Yanofsky, M. F. (2000a). Redundant regulation of meristem identity and plant architecture by FRUITFULL, APETALA1 and CAULIFLOWER. Development 127 (4), 725-734.

Ferrandiz, C., Liljegren, S. J., and Yanofsky, M. F. (2000b). Negative regulation of the SHATTERPROOF genes by FRUITFULL during Arabidopsis fruit development. Science 289 (5478), 436-438. doi: 10.1126/science.289.5478.436

Franco-Zorrilla, J. M., Valli, A., Todesco, M., Mateos, I., Puga, M. I., Rubio-Somoza, I., et al. (2007). Target mimicry provides a new mechanism for regulation of microRNA activity. Nat. Genet. 39 (8), 1033-1037. doi: 10.1038/ng2079

Freese, N. H., Norris, D. C., and Loraine, A. E. (2016). Integrated genome browser: visual analytics platform for genomics. Bioinformatics 32 (14), 2089-2095. doi: 10.1093/bioinformatics/btw069

Gan, Y., Filleur, S., Rahman, A., Gotensparre, S., and Forde, B. G. (2005). Nutritional regulation of ANR1 and other root-expressed MADS-box genes in Arabidopsis thaliana. Planta 222 (4), 730-742. doi: 10.1007/s00425-005-0020-3

Gantier, M. P., McCoy, C. E., Rusinova, I., Saulep, D., Wang, D., Xu, D., et al. (2011). Analysis of microRNA turnover in mammalian cells following Dicer1 ablation. Nucleic Acids Res. 39 (13), 5692-5703. doi: 10.1093/nar/gkr148

Geraldo, N., Baurle, I., Kidou, S., Hu, X., and Dean, C. (2009). FRIGIDA delays flowering in Arabidopsis via a cotranscriptional mechanism involving direct interaction with the nuclear cap-binding complex. Plant Physiol. 150 (3), 16111618. doi: $10.1104 /$ pp.109.137448

Gramzow, L., and Theissen, G. (2015). Phylogenomics reveals surprising sets of essential and dispensable clades of MIKC(c)-group MADS-box genes in flowering plants. J. Exp. Zool B Mol. Dev. Evol. 324 (4), 353-362. doi: 10.1002/jez.b.22598

Gregory, B. D., O'Malley, R. C., Lister, R., Urich, M. A., Tonti-Filippini, J., Chen, H., et al. (2008). A link between RNA metabolism and silencing affecting Arabidopsis development. Dev. Cell 14 (6), 854-866. doi: 10.1016/J. Devcel.2008.04.005

Guan, Q., Lu, X., Zeng, H., Zhang, Y., and Zhu, J. (2013). Heat stress induction of miR398 triggers a regulatory loop that is critical for thermotolerance in Arabidopsis. Plant J. 74 (5), 840-851. doi: 10.1111/tpj.12169

Gy, I., Gasciolli, V., Lauressergues, D., Morel, J. B., Gombert, J., Proux, F., et al. (2007). Arabidopsis FIERY1, XRN2, and XRN3 are endogenous RNA silencing suppressors. Plant Cell 19 (11), 3451-3461. doi: 10.1105/Tpc.107.055319

Gyula, P., Baksa, I., Toth, T., Mohorianu, I., Dalmay, T., and Szittya, G. (2018). Ambient temperature regulates the expression of a small set of sRNAs influencing plant development through NF-YA2 and YUC2. Plant Cell Environ. 41 (10), 2404-2417. doi: 10.1111/pce.13355

Halbach, F., Reichelt, P., Rode, M., and Conti, E. (2013). The yeast ski complex: crystal structure and RNA channeling to the exosome complex. Cell 154 (4), 814-826. doi: 10.1016/j.cell.2013.07.017

He, F., Xu, C., Fu, X., Shen, Y., Guo, L., Leng, M., et al. (2018). The microRNA390/ trans acting short interfering RNA 3 module mediates lateral root growth under salt stress via the auxin pathway. Plant Physiol. 177 (2), 775-791. doi: 10.1104/pp.17.01559

Hepworth, J., Antoniou-Kourounioti, R. L., Bloomer, R. H., Selga, C., Berggren, K., Cox, D., et al. (2018). Absence of warmth permits epigenetic memory of winter in Arabidopsis. Nat. Commun. 9 (1), 639. doi: 10.1038/s41467-018-03065-7

Hu, J. Y., Zhou, Y., He, F., Dong, X., Liu, L. Y., Coupland, G., et al. (2014). miR824Regulated AGAMOUS-LIKE16 Contributes to Flowering Time Repression in Arabidopsis. Plant Cell 26 (5), 2024-2037. doi: 10.1105/tpc.114.124685 
Huang, Y. C., Niu, C. Y., Yang, C. R., and Jinn, T. L. (2016). The Heat Stress Factor HSFA6b Connects ABA Signaling and ABA-Mediated Heat Responses. Plant Physiol. 172 (2), 1182-1199. doi: 10.1104/pp.16.00860

Hwang, S. M., Kim, D. W., Woo, M. S., Jeong, H. S., Son, Y. S., Akhter, S., et al. (2014). Functional characterization of Arabidopsis HsfA6a as a heat-shock transcription factor under high salinity and dehydration conditions. Plant Cell Environ. 37 (5), 1202-1222. doi: 10.1111/pce.12228

Kim, D., Langmead, B., and Salzberg, S. L. (2015). HISAT: a fast spliced aligner with low memory requirements. Nat. Methods 12 (4), 357-360. doi: 10.1038/ nmeth. 3317

Kobayashi, Y., and Weigel, D. (2007). Move on up, it's time for change-mobile signals controlling photoperiod-dependent flowering. Genes Dev. 21 (19), 2371-2384. doi: 10.1101/gad.1589007

Kruszka, K., Pacak, A., Swida-Barteczka, A., Nuc, P., Alaba, S., Wroblewska, Z., et al. (2014). Transcriptionally and post-transcriptionally regulated microRNAs in heat stress response in barley. J. Exp. Bot. 65 (20), 6123-6135. doi: 10.1093/ jxb/eru353

Kumar, R. (2014). Role of microRNAs in biotic and abiotic stress responses in crop plants. Appl. Biochem. Biotechnol. 174 (1), 93-115. doi: 10.1007/ s12010-014-0914-2

Kumar, R. R., Pathak, H., Sharma, S. K., Kala, Y. K., Nirjal, M. K., Singh, G. P., et al. (2015). Novel and conserved heat-responsive microRNAs in wheat (Triticum aestivum L.). Funct. Integr. Genomics 15 (3), 323-348. doi: 10.1007/ s10142-014-0421-0

Kutter, C., Schob, H., Stadler, M., Meins, F., and Si-Ammour, A. (2007). MicroRNAmediated regulation of stomatal development in Arabidopsis. Plant Cell 19 (8), 2417-2429. doi: 10.1105/tpc.107.050377

Lamesch, P., Berardini, T. Z., Li, D., Swarbreck, D., Wilks, C., Sasidharan, R., et al. (2012). The Arabidopsis Information Resource (TAIR): improved gene annotation and new tools. Nucleic Acids Res. 40, D1202-D1210. doi: 10.1093/ nar/gkr1090

Lamke, J., and Baurle, I. (2017). Epigenetic and chromatin-based mechanisms in environmental stress adaptation and stress memory in plants. Genome Biol. 18 (1), 124. doi: 10.1186/s13059-017-1263-6

Lamke, J., Brzezinka, K., Altmann, S., and Baurle, I. (2016). A hit-and-run heat shock factor governs sustained histone methylation and transcriptional stress memory. EMBO J. 35 (2), 162-175. doi: 10.15252/embj.201592593

Lee, I., and Amasino, R. M. (1995). Effect of Vernalization, Photoperiod, and Light Quality on the Flowering Phenotype of Arabidopsis Plants Containing the FRIGIDA Gene. Plant Physiol. 108 (1), 157-162. doi: 10.1104/pp.108.1.157

Li, C., and Zhang, B. (2016). MicroRNAs in Control of Plant Development. J. Cell Physiol. 231 (2), 303-313. doi: 10.1002/jcp.25125

Li, H., Handsaker, B., Wysoker, A., Fennell, T., Ruan, J., Homer, N., et al. (2009). The Sequence Alignment/Map format and SAMtools. Bioinformatics 25 (16), 2078-2079. doi: 10.1093/bioinformatics/btp352

Liljegren, S. J., Ditta, G. S., Eshed, Y., Savidge, B., Bowman, J. L., and Yanofsky, M. F. (2000). SHATTERPROOF MADS-box genes control seed dispersal in Arabidopsis. Nature 404 (6779), 766-770. doi: 10.1038/35008089

Lin, J. S., Kuo, C. C., Yang, I. C., Tsai, W. A., Shen, Y. H., Lin, C. C., et al. (2018). MicroRNA160 Modulates Plant Development and Heat Shock Protein Gene Expression to Mediate Heat Tolerance in Arabidopsis. Front. Plant Sci. 9, 68. doi: $10.3389 /$ fpls.2018.00068

Ling, Y., Serrano, N., Gao, G., Atia, M., Mokhtar, M., Woo, Y. H., et al. (2018). Thermopriming triggers splicing memory in Arabidopsis. J. Exp. Bot. 69 (10), 2659-2675. doi: 10.1093/jxb/ery062

Liu, H. C., and Charng, Y. Y. (2012). Acquired thermotolerance independent of heat shock factor A1 (HsfA1), the master regulator of the heat stress response. Plant Signal Behav. 7 (5), 547-550. doi: 10.4161/psb.19803

Liu, H. C., and Charng, Y. Y. (2013). Common and distinct functions of Arabidopsis class A1 and A2 heat shock factors in diverse abiotic stress responses and development. Plant Physiol. 163 (1), 276-290. doi: 10.1104/pp.113.221168

Liu, H. C., Lamke, J., Lin, S. Y., Hung, M. J., Liu, K. M., Charng, Y. Y., et al. (2018). Distinct heat shock factors and chromatin modifications mediate the organautonomous transcriptional memory of heat stress. Plant J. 95 (3), 401-413. doi: $10.1111 /$ tpj. 13958

Liu, H. C., Liao, H. T., and Charng, Y. Y. (2011). The role of class A1 heat shock factors (HSFA1s) in response to heat and other stresses in Arabidopsis. Plant Cell Environ. 34 (5), 738-751. doi: 10.1111/j.1365-3040.2011.02278.x
Liu, J., Feng, L., Gu, X., Deng, X., Qiu, Q., Li, Q., et al. (2019). An H3K27me3 demethylase-HSFA2 regulatory loop orchestrates transgenerational thermomemory in Arabidopsis. Cell Res. 29 (5), 379-390. doi: 10.1038/ s41422-019-0145-8

Liu, W., Xu, L., Wang, Y., Shen, H., Zhu, X., Zhang, K., et al. (2015). Transcriptomewide analysis of chromium-stress responsive microRNAs to explore miRNAmediated regulatory networks in radish (Raphanus sativus L.). Sci. Rep. 5, 14024. doi: 10.1038/srep14024

Lozano, R., Angosto, T., Gomez, P., Payan, C., Capel, J., Huijser, P., et al. (1998). Tomato flower abnormalities induced by low temperatures are associated with changes of expression of MADS-Box genes. Plant Physiol. 117 (1), 91-100. doi: 10.1104/pp.117.1.91

Luo, Y., Guo, Z., and Li, L. (2013). Evolutionary conservation of microRNA regulatory programs in plant flower development. Dev. Biol. 380 (2), 133-144. doi: 10.1016/j.ydbio.2013.05.009

Ma, C., Burd, S., and Lers, A. (2015). miR408 is involved in abiotic stress responses in Arabidopsis. Plant J. 84 (1), 169-187. doi: 10.1111/tpj.12999

Mangrauthia, S. K., Bhogireddy, S., Agarwal, S., Prasanth, V. V., Voleti, S. R., Neelamraju, S., et al. (2017). Genome-wide changes in microRNA expression during short and prolonged heat stress and recovery in contrasting rice cultivars. J. Exp. Bot. 68 (9), 2399-2412. doi: 10.1093/jxb/erx111

Matthewman, C. A., Kawashima, C. G., Huska, D., Csorba, T., Dalmay, T., and Kopriva, S. (2012). miR395 is a general component of the sulfate assimilation regulatory network in Arabidopsis. FEBS Lett. 586 (19), 3242-3248. doi: 10.1016/j.febslet.2012.06.044

McClung, C. R., Lou, P., Hermand, V., and Kim, J. A. (2016). The Importance of Ambient Temperature to Growth and the Induction of Flowering. Front. Plant Sci. 7, 1266. doi: 10.3389/fpls.2016.01266

Medzihradszky, A., Schneitz, K., and Lohmann, J. U. (2014). Detection of mRNA expression patterns by nonradioactive in situ hybridization on histological sections of floral tissue. Methods Mol. Biol. 1110, 275-293. doi: 10.1007/978-1-4614-9408-9_14

Meiri, D., and Breiman, A. (2009). Arabidopsis ROF1 (FKBP62) modulates thermotolerance by interacting with HSP90.1 and affecting the accumulation of HsfA2-regulated sHSPs. Plant J. 59 (3), 387-399. doi: 10.1111/j.1365-313X.2009.03878.x

Merret, R., Descombin, J., Juan, Y. T., Favory, J. J., Carpentier, M. C., Chaparro, C., et al. (2013). XRN4 and LARP1 are required for a heat-triggered mRNA decay pathway involved in plant acclimation and survival during thermal stress. Cell Rep. 5 (5), 1279-1293. doi: 10.1016/j.celrep.2013.11.019

Merret, R., Nagarajan, V. K., Carpentier, M. C., Park, S., Favory, J. J., Descombin, J., et al. (2015). Heat-induced ribosome pausing triggers mRNA co-translational decay in Arabidopsis thaliana. Nucleic Acids Res. 43 (8), 4121-4132. doi: 10.1093/nar/gkv234

Messenguy, F., and Dubois, E. (2003). Role of MADS box proteins and their cofactors in combinatorial control of gene expression and cell development. Gene 316, 1-21. doi: 10.1016/s0378-1119(03)00747-9

Michaels, S. D., and Amasino, R. M. (1999). FLOWERING LOCUS C encodes a novel MADS domain protein that acts as a repressor of flowering. Plant Cell 11 (5), 949-956. doi: 10.1105/tpc.11.5.949

Michaels, S. D., Ditta, G., Gustafson-Brown, C., Pelaz, S., Yanofsky, M., and Amasino, R. M. (2003). AGL24 acts as a promoter of flowering in Arabidopsis and is positively regulated by vernalization. Plant J. 33 (5), 867-874. doi: 10.1046/j.1365-313x.2003.01671.x

Mittler, R., Finka, A., and Goloubinoff, P. (2012). How do plants feel the heat? Trends Biochem. Sci. 37 (3), 118-125. doi: 10.1016/j.tibs.2011.11.007

Nesi, N., Debeaujon, I., Jond, C., Stewart, A. J., Jenkins, G. I., Caboche, M., et al. (2002). The TRANSPARENT TESTA16 locus encodes the ARABIDOPSIS BSISTER MADS domain protein and is required for proper development and pigmentation of the seed coat. Plant Cell 14 (10), 2463-2479. doi: 10.1105/tpc.004127

Nie, S. S., Xu, L., Wang, Y., Huang, D. Q., Muleke, E. M., Sun, X. C., et al. (2015). Identification of bolting-related microRNAs and their targets reveals complex miRNA-mediated flowering-time regulatory networks in radish (Raphanus sativus L.). Sci. Rep. 5, 14034. doi: 10.1038/srep14034

Nishizawa-Yokoi, A., Nosaka, R., Hayashi, H., Tainaka, H., Maruta, T., Tamoi, M., et al. (2011). HsfAld and HsfAle involved in the transcriptional regulation of HsfA2 function as key regulators for the Hsf signaling network in response to environmental stress. Plant Cell Physiol. 52 (5), 933-945. doi: 10.1093/pcp/pcr045 
Pelaz, S., Ditta, G. S., Baumann, E., Wisman, E., and Yanofsky, M. F. (2000). B and $\mathrm{C}$ floral organ identity functions require SEPALLATA MADS-box genes. Nature 405 (6783), 200-203. doi: 10.1038/35012103

Ramirez, F., Ryan, D. P., Gruning, B., Bhardwaj, V., Kilpert, F., Richter, A. S., et al. (2016). Deeptools2: a next generation web server for deep-sequencing data analysis. Nucleic Acids Res. 44 (W1), W160-W165. doi: 10.1093/nar/gkw257

Rogers, K., and Chen, X. (2013). Biogenesis, turnover, and mode of action of plant microRNAs. Plant Cell 25 (7), 2383-2399. doi: 10.1105/tpc.113.113159

Romera-Branchat, M., Andres, F., and Coupland, G. (2014). Flowering responses to seasonal cues: what's new? Curr. Opin. Plant Biol. 21, 120-127. doi: 10.1016/j. pbi.2014.07.006

Rubio-Somoza, I., and Weigel, D. (2011). MicroRNA networks and developmental plasticity in plants. Trends Plant Sci. 16 (5), 258-264. doi: 10.1016/j. tplants.2011.03.001

Saha, G., Park, J. I., Jung, H. J., Ahmed, N. U., Kayum, M. A., Chung, M. Y., et al. (2015). Genome-wide identification and characterization of MADS-box family genes related to organ development and stress resistance in Brassica rapa. BMC Genomics 16, 178. doi: 10.1186/s12864-015-1349-z

Sanei, M., and Chen, X. (2015). Mechanisms of microRNA turnover. Curr. Opin. Plant Biol. 27, 199-206. doi: 10.1016/j.pbi.2015.07.008

Scharf, K. D., Berberich, T., Ebersberger, I., and Nover, L. (2012). The plant heat stress transcription factor (Hsf) family: structure, function and evolution. Biochim. Biophys. Acta 1819 (2), 104-119. doi: 10.1016/j.bbagrm.2011.10.002

Schommer, C., Palatnik, J. F., Aggarwal, P., Chetelat, A., Cubas, P., Farmer, E. E., et al. (2008). Control of jasmonate biosynthesis and senescence by miR319 targets. PloS Biol. 6 (9), e230. doi: 10.1371/journal.pbio.0060230

Schramm, F., Larkindale, J., Kiehlmann, E., Ganguli, A., Englich, G., Vierling, E., et al. (2008). A cascade of transcription factor DREB2A and heat stress transcription factor HsfA3 regulates the heat stress response of Arabidopsis. Plant J. 53 (2), 264-274. doi: 10.1111/j.1365-313X.2007.03334.x

Silhavy, D., Molnar, A., Lucioli, A., Szittya, G., Hornyik, C., Tavazza, M., et al. (2002). A viral protein suppresses RNA silencing and binds silencing-generated, 21- to 25-nucleotide double-stranded RNAs. EMBO J. 21 (12), 3070-3080. doi: 10.1093/emboj/cdf312

Smaczniak, C., Immink, R. G., Angenent, G. C., and Kaufmann, K. (2012). Developmental and evolutionary diversity of plant MADS-domain factors: insights from recent studies. Development 139 (17), 3081-3098. doi: 10.1242/ dev.074674

Souret, F. F., Kastenmayer, J. P., and Green, P. J. (2004). AtXRN4 degrades mRNA in Arabidopsis and its substrates include selected miRNA targets. Mol. Cell 15 (2), 173-183. doi: 10.1016/j.molcel.2004.06.006

Srinivasan, R., Daniels, J., Fusaro, V., Lundqvist, A., Killian, J. K., Geho, D., et al. (2006). Accurate diagnosis of acute graft-versus-host disease using serum proteomic pattern analysis. Exp. Hematol. 34 (6), 796-801. doi: 10.1016/j. exphem.2006.02.013

Stief, A., Altmann, S., Hoffmann, K., Pant, B. D., Scheible, W. R., and Baurle, I. (2014). Arabidopsis miR156 Regulates Tolerance to Recurring Environmental Stress through SPL Transcription Factors. Plant Cell 26 (4), 1792-1807. doi: 10.1105/tpc.114.123851

Sunkar, R., Li, Y. F., and Jagadeeswaran, G. (2012). Functions of microRNAs in plant stress responses. Trends Plant Sci. 17 (4), 196-203. doi: 10.1016/j. tplants.2012.01.010

Szadeczky-Kardoss, I., Csorba, T., Auber, A., Schamberger, A., Nyiko, T., Taller, J., et al. (2018). The nonstop decay and the RNA silencing systems operate cooperatively in plants. Nucleic Acids Res. 46 (9), 4632-4648. doi: 10.1093/nar/ gky279

Takada, S., and Goto, K. (2003). TERMINAL FLOWER2, an Arabidopsis homolog of HETEROCHROMATIN PROTEIN1, counteracts the activation of FLOWERING LOCUS T by CONSTANS in the vascular tissues of leaves to regulate flowering time. Plant Cell 15 (12), 2856-2865. doi: 10.1105/ tpc. 016345
Tao, Z., Shen, L., Liu, C., Liu, L., Yan, Y., and Yu, H. (2012). Genome-wide identification of SOC1 and SVP targets during the floral transition in Arabidopsis. Plant J. 70 (4), 549-561. doi: 10.1111/j.1365-313X.2012.04919.x

Tardif, G., Kane, N. A., Adam, H., Labrie, L., Major, G., Gulick, P., etal. (2007). Interaction network of proteins associated with abiotic stress response and development in wheat. Plant Mol. Biol. 63 (5), 703-718. doi: 10.1007/s11103-006-9119-6

van Hoof, A., Frischmeyer, P.A., Dietz, H.C., and Parker, R. (2002). Exosomemediated recognition and degradation of mRNAs lacking a termination codon. Science 295 (5563), 2262-2264. doi: 10.1126/science.1067272

Wang, J. W., Czech, B., and Weigel, D. (2009). miR156-regulated SPL transcription factors define an endogenous flowering pathway in Arabidopsis thaliana. Cell 138 (4), 738-749. doi: 10.1016/j.cell.2009.06.014

Whittaker, C., and Dean, C. (2017). The FLC Locus: A Platform for Discoveries in Epigenetics and Adaptation. Annu. Rev. Cell Dev. Biol. 33, 555-575. doi: 10.1146/annurev-cellbio-100616-060546

Wu, D., Liang, Z., Yan, T., Xu, Y., Xuan, L., Tang, J., et al. (2019). Whole-Genome Resequencing of a Worldwide Collection of Rapeseed Accessions Reveals the Genetic Basis of Ecotype Divergence. Mol. Plant 12 (1), 30-43. doi: 10.1016/j. molp.2018.11.007

Wu, T. Y., Juan, Y. T., Hsu, Y. H., Wu, S. H., Liao, H. T., Fung, R. W., et al. (2013). Interplay between heat shock proteins, HSP101 and HSA32, prolongs heat acclimation memory posttranscriptionally in Arabidopsis. Plant Physiol. 161 (4), 2075-2084. doi: 10.1104/pp.112.212589

Xin, M., Wang, Y., Yao, Y., Song, N., Hu, Z., Qin, D., et al. (2011). Identification and characterization of wheat long non-protein coding RNAs responsive to powdery mildew infection and heat stress by using microarray analysis and SBS sequencing. BMC Plant Biol. 11, 61. doi: 10.1186/1471-2229-11-61

Yang, K., Jiang, M., and Le, J. (2014). A new loss-of-function allele 28y reveals a role of ARGONAUTE1 in limiting asymmetric division of stomatal lineage ground cell. J. Integr. Plant Biol. 56 (6), 539-549. doi: 10.1111/jipb.12154

Yanofsky, M. F., Ma, H., Bowman, J. L., Drews, G. N., Feldmann, K. A., and Meyerowitz, E. M. (1990). The protein encoded by the Arabidopsis homeotic gene agamous resembles transcription factors. Nature 346 (6279), 35-39. doi: $10.1038 / 346035 \mathrm{a} 0$

Yeh, C. H., Kaplinsky, N. J., Hu, C., and Charng, Y. Y. (2012). Some like it hot, some like it warm: phenotyping to explore thermotolerance diversity. Plant Sci. 195, 10-23. doi: 10.1016/j.plantsci.2012.06.004

Yoshida, T., Ohama, N., Nakajima, J., Kidokoro, S., Mizoi, J., Nakashima, K., et al. (2011). Arabidopsis HsfA1 transcription factors function as the main positive regulators in heat shock-responsive gene expression. Mol. Genet. Genomics 286 (5-6), 321-332. doi: 10.1007/s00438-011-0647-7

Yu, X., Wang, H., Lu, Y., de Ruiter, M., Cariaso, M., Prins, M., et al. (2012). Identification of conserved and novel microRNAs that are responsive to heat stress in Brassica rapa. J. Exp. Bot. 63 (2), 1025-1038. doi: 10.1093/jxb/err337

Zhang, B. (2015). MicroRNA: a new target for improving plant tolerance to abiotic stress. J. Exp. Bot. 66 (7), 1749-1761. doi: 10.1093/jxb/erv013

Zhang, T., Marand, A. P., and Jiang, J. (2016). PlantDHS: a database for DNase I hypersensitive sites in plants. Nucleic Acids Res. 44 (D1), D1148-D1153. doi: 10.1093/nar/gkv962

Conflict of Interest: The authors declare that the research was conducted in the absence of any commercial or financial relationships that could be construed as a potential conflict of interest.

Copyright (C) 2019 Szaker, Darkó, Medzihradszky, Janda, Liu, Charng and Csorba. This is an open-access article distributed under the terms of the Creative Commons Attribution License (CC BY). The use, distribution or reproduction in other forums is permitted, provided the original author(s) and the copyright owner(s) are credited and that the original publication in this journal is cited, in accordance with accepted academic practice. No use, distribution or reproduction is permitted which does not comply with these terms. 